\title{
Interhemispheric Cortico-Cortical Pathway for Sequential Bimanual Movements in Mice
}

\author{
Minju Jeong, ${ }^{1,4, *}$ - $H y e o n s u$ Lee, ${ }^{2, *}$ ○Youngsoo Kim, ${ }^{1}$ Eric Hou-Jen Wang, ${ }^{5}$ 'Se-Bum Paik, ${ }^{2,3}$ \\ Byung Kook Lim, ${ }^{4,5}$ and ${ }^{-D a e s o o ~} \mathrm{Kim}^{1}$
}

\section{https://doi.org/10.1523/ENEURO.0200-21.2021}

\begin{abstract}
${ }^{1}$ Department of Biological Sciences, Korea Advanced Institute of Science and Technology, Daejeon 34141, Republic of Korea, ${ }^{2}$ Department of Bio and Brain Engineering, Korea Advanced Institute of Science and Technology, Daejeon 34141, Republic of Korea, ${ }^{3}$ Program of Brain and Cognitive Engineering, Korea Advanced Institute of Science and Technology, Daejeon 34141, Republic of Korea, ${ }^{4}$ Neurobiology Section, Division of Biological Sciences, University of California, San Diego, La Jolla, CA 92093, and ${ }^{5}$ Biomedical Sciences Graduate Program, University of California, San Diego, La Jolla, CA 92093
\end{abstract}

\begin{abstract}
Animals precisely coordinate their left and right limbs for various adaptive purposes. While the left and right limbs are clearly controlled by different cortical hemispheres, the neural mechanisms that determine the action sequence between them remains elusive. Here, we have established a novel head-fixed bimanual-press (biPress) sequence task in which mice sequentially press left and right pedals with their forelimbs in a predetermined order. Using this motor task, we found that the motor cortical neurons responsible for the first press (1P) also generate independent motor signals for the second press (2P) by the opposite forelimb during the movement transitions between forelimbs. Projection-specific calcium imaging and optogenetic manipulation revealed these motor signals are transferred from one motor cortical hemisphere to the other via corticocortical projections. Together, our results suggest the motor cortices coordinate sequential bimanual movements through corticocortical pathways.
\end{abstract}

Key words: cortico-cortical pathway; interhemispheric projection; sequential bimanual movements

\section{Significance Statement}

The orchestration of left and right limbs is required to perform complex behaviors in our daily life. It has been presumed that the two cortical hemispheres might interact with each other through the callosal pathways. Yet, the exact neural circuit mechanisms how information exchange of limb movements occurs between the two hemispheres still remain unclear. Here, we establish a novel motor task in head-fixed mice to perform sequentially press left and right pedals, respectively. We then found out that the transfer of motor signals via corticocortical pathways arisen during the movement transition period is essential for precise coordination of left and right limbs. Our findings implicate the functional roles of corticocortical pathways in complex motor behaviors.

\section{Introduction}

The intricate coordination between our limbs underlies much of our daily tasks, performing musical instruments, playing sports, or manipulating various objects. Specifically, the left and right limbs need to be elaborately coordinated,

Received May 5, 2021; accepted July 28, 2021; First published August 4, 2021.

The authors declare no competing financial interests. with smooth transition between action sequences and timing (Lashley, 1951; Diedrichsen and Kornysheva, 2015), to perform complex behaviors. Although it is well established that each hemisphere of the mammalian brain can control the opposite limb movements (Penfield and Rasmussen,

Author contributions: D.K., and M.J. designed research; M.J., Y.K., and E.H.-J.W. performed research; B.K.L., S.-B.P., and H.L. contributed unpublished reagents/analytic tools; M.J. and H.L. analyzed data; D.K., M.J., H.L., E.H.-J.W., S.-B.P., and B.K.L. wrote the paper. 
1950; Tanji et al., 1988; Lemon, 2008), it remains unclear how these hemispheres interact to coordinate temporally precise movements that require both left and right limbs.

Previous observations have reported that patients with a damaged callosal pathway showing symptoms of aberrant spatiotemporal coordination between left and right hands (Eliassen et al., 1999; Kennerley et al., 2002; Bonzano et al., 2008), suggesting that the callosal pathway seems to be important for bimanual coordination (Bloom and Hynd, 2005; Gooijers and Swinnen, 2014). As the callosum is a major pathway for interhemispheric corticocortical connectivity (Wahl et al., 2007; Zhou et al., 2013; Oh et al., 2014; Zingg et al., 2014), the prevailing assumption, yet uncharacterized, is that interhemispheric corticocortical pathways regulate these bimanual movements.

Although recent developments in neuro-imaging, optogenetics, and behavioral measurements have advanced our understanding of the neural substrate of unilateral movements in rodents (Makino et al., 2017; Terada et al., 2018; Mayrhofer et al., 2019), we still understand little about the regulation of sequential bimanual movements. This is, in part, because of a lack of sophisticated behavioral measurements that can reliably constrain sequential bimanual movements. Addressing this problem required us to develop a novel head-fixed, behavioral paradigm that trains mice to sequentially produce, temporally precise bimanual movements. By combining this approach with calcium imaging, in vivo extracellular recording, projection-specific viral targeting, and optogenetics, we found that sequential transfer of motor signals through interhemispheric corticocortical projections contribute to the sequential bimanual movements.

\section{Materials and Methods}

\section{Animals}

All animal care and experimental procedures were performed in accordance with protocols approved by the directives of the Animal Care and Use Committee of Korea Advanced Institute of Science and Technology (approval number KA2012-04) and the Institutional Animal Care and Use Committee (IACUC) of the University of California, San Diego. Either of male or female C57BL/6J, VGATChR2 (JAX 014548), and Thy1-GCaMP6s (JAX 024275) mice were used. All mice applied for behavioral tests were

This work was supported by the Samsung Science and Technology Foundation Grant SSTF-BA1301-53 (to D.K.). M.J. was supported by the National Research Foundation of Korea (NRF) Fellowship 2018R1A6A3A11050549. B.K.L. and E.H.-J. W. were supported by National Institutes of Health Grant R01 MH107742, R01 ND097772, and R21 NS114610. H.L. and S.-B.P. were supported by NRF Grants 2019R1A2C4069863 and 2019M3E5D2A01058328.

${ }^{*} M . J$. and H.L. contributed equally to this work.

Correspondence should be addressed to Se-Bum Paik at sbpaik@ kaist.ac.kr, Byung Kook Lim at bklim@ucsd.edu, or Daesoo Kim at daesoo@kaist.ac.kr.

https://doi.org/10.1523/ENEURO.0200-21.2021

Copyright (C) 2021 Jeong, Lee et al.

This is an open-access article distributed under the terms of the Creative Commons Attribution 4.0 International license, which permits unrestricted use, distribution and reproduction in any medium provided that the original work is properly attributed. single-housed under a 12/12 h light/dark cycle with free access to rodent chow, with water restriction initiated after the head-implant surgery.

\section{Surgery}

Mice were anesthetized with 2,2,2-tribromoethanol $(20 \mathrm{mg} / \mathrm{ml}$, i.p.; Sigma T48402) or $1-2 \%$ isoflurane and placed on a stereotaxic apparatus (Kopf Instruments). For wide-field imaging (WFI) experiments, the skulls of Thy1:: GCaMP6s mice were thinned to $\sim 250-500 \mu \mathrm{m}$ thick using a dental drill, to obtain high quality images of the cortical surface. Thereafter, a custom-designed head plate was implanted with dental cement (C\&B Metabond; Sun Medical Co or Parkell). For in vivo single-unit recording experiments, a head plate was implanted to the skull of each C57BL/6J mouse. After the cement hardened, the opened skull was covered with Kwik-Sil (WPI) for protection. For GCaMP photometry experiments, $0.5 \mu$ of premixed AAV9-CamKIIO.4-Cre-SV4O $\left(3.87 \times 10^{13}\right.$ particles $/ \mathrm{ml}$; University of Pennsylvania) and AAV9-CAG-Flex-GCaMP6fWPRE-SV40 $\left(2.61 \times 10^{13}\right.$ particles $/ \mathrm{ml}$; University of Pennsylvania; $1: 1$ ratio) viruses were injected into the caudal forelimb area (CFA; AP + 0.85; $\mathrm{ML}-1.1$; DV -1.2 mm) of the C57BL/6J mice. After the viral injection, an optic cannula (F1.5-MM-FP-OPTH; Becker \& Hickl GmbH) was implanted into the opposite-hemisphere CFA (AP + 0.85; $\mathrm{ML}+1.1$; DV $-1.0 \mathrm{~mm}$ ) and a head plate was implanted to the skull. For optogenetic experiments, an optic cannula (MFC_200/230-0.48_1.3 mm MF1.25_FLT; Doric Lenses Inc.) was inserted into the CFA and a head plate was implanted onto the skull of VGAT::ChR2 and littermate control mice. For optogenetic inhibition of corticocortical afferents, $0.5 \mu$ of retroAAVDJ-Cre and AAVDJ-EF1a-Flex-ArchT-GFP were injected into each CFA of C57BL/6J mice. These two adeno-associated virus (AAV) vectors were generated as previously described (Lim et al., 2012). After viral injection, the optic cannula was implanted into the CFA of the hemisphere that had been injected with the retroAAVDJ-Cre viruses, and a head plate was implanted onto the skull.

\section{Bimanual-press (biPress) sequence task training}

The experimental apparatus was custom-made with a 3D printer (Marv; Canon, Ultimaker S2; Ultimaker) and the software for manipulating the hardware was custom-designed using the Arduino program (Mega2560 boards; Arduino). Water was delivered by a peristaltic pump. The pedal-press state and time were recorded by a customdesigned Python program. When a mouse pushes the pedal down with enough force, it flips a microswitch under the pedal, an Arduino-controlled step motor rotates to lock the pedals into position (Extended Data Fig. 1-1). After each trial, the step motor rotates back in the opposite direction, resetting the retracted pedals. We designed this apparatus in which a mouse cannot re-press the pedal until it is reset to avoid the confounding from double-taps.

At $3 \sim 5 \mathrm{~d}$ after the surgery, mice were water restricted to $1 \mathrm{ml} / \mathrm{d}$ for the training, which proceeded via three stages. During stage 1 of the training $(<7 d)$, the mice 
A

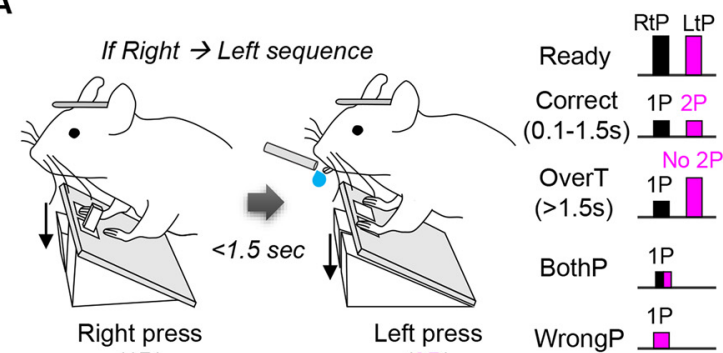

(1P)

C

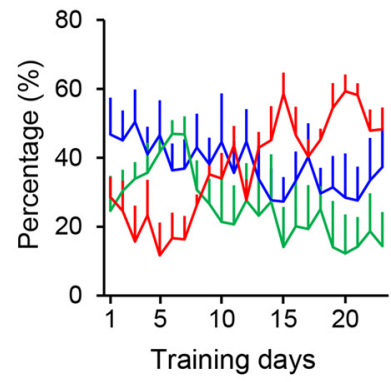

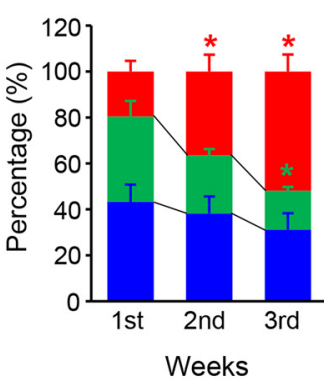

Weeks

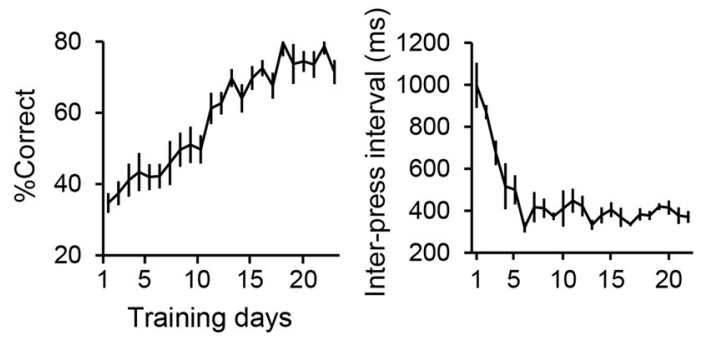

D
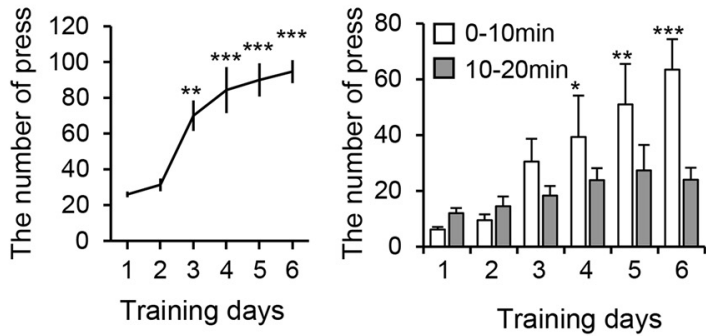

Figure 1. biPress sequence task training. $\boldsymbol{A}$, Schematic diagram of the biPress motor task. Left, long bars and short bars represent non-pressed and pressed state, respectively. Black and magenta represent 1P and 2P (see also Extended Data Fig. 1-1 for description of pedal movements in each trial). $\boldsymbol{B}$, left, Behavior performance represented as the percentage of correct trials. Right, The time interval between the $1 \mathrm{P}$ and the $2 \mathrm{P}$. $\boldsymbol{C}$, left, The percentage of each incorrect trial over the total number of incorrect trials during the training period. Right, The fraction of each incorrect trial per week during the training period. $\boldsymbol{D}$, The number of pedal-press during stage 1 of the biPress task training. Left, The total number of pedal-press in a whole session. Right, The number of pedal-press early (0-10 min) and late (10-20 min) phase of a daily session. All data are represented as mean $\pm \mathrm{SEM}$; ${ }^{*} p<0.05,{ }^{\star *} p<0.01,{ }^{\star \star *} p<0.001$.

Figure Contributions: Minju Jeong performed the experiments and analyzed the data.

were water-rewarded for singly pressing the left or right pedal, but not for simultaneously pressing both pedals. The mice were trained only to press the pedals, not to hold them down. Once a mouse exceeded 70 correct trials within $20 \mathrm{~min}$ [ $n=6$ mice, one-way RM ANOVA with Tukey's post hoc test, $F_{(5,35)}=19.127, p<0.001$, $p=0.002$ for day $3, p<0.001$ for days $4-6$ compared with day 1 (Fig. $1 D$, left); two-way RM ANOVA, days: $F_{(5,71)}=$ 10.974, $p<0.001$ (Fig. 1D, right)], it advanced to the next stage. In stage 2 of the training (2d), mice were rewarded for pressing either the left $\rightarrow$ right or right $\rightarrow$ left sequence. If a mouse simultaneously pressed both pedals, no water reward was given. Once a mouse exceeded 70 correct trials within $20 \mathrm{~min}$, it advanced to the next stage. We excluded the mice that did not learn the both left and right pedal-press from the next stage. In the final stage of the training $(>21 \mathrm{~d})$, the pressing sequence was fixed in one direction. The mice were rewarded for pressing each pedal in the desired sequence within 5-1.5 s. This between-pedal-press interval (1-2P period) was gradually decreased by $0.5 \mathrm{~s}$ for every 10 correct trials. If a mouse pressed both pedals at the same time or pressed the wrong side first, the event was regarded as an incorrect trial (bothP and wrongP, respectively). If the mouse pressed the correct-side pedal at first but did not press the other side pedal within the desired interval, it was also regarded as an incorrect trial (overtime fail, overT). A session was conducted for $15 \mathrm{~min} / \mathrm{d}$. All trials were voluntarily started without any sensory cue.

\section{Timed unimanual-press (uniPress) sequence task training}

The same experimental apparatus was used as the biPress motor task. Three to $5 \mathrm{~d}$ after the surgery, mice were water restricted to $1 \mathrm{ml} / \mathrm{d}$ for the training. During the first $5 \mathrm{~d}$, the mice were trained to press the pedal, in which they were water-rewarded for singly pressing either the left or right pedal. Reward-associated pedal was assigned by the location of viral injection, which was either ipsilateral or contralateral to axon terminals of corticocortical projections (Fig. 4G). After the single pedal-press, an Arduino-controlled step motor rotates to lock the pedals into position, and it rotates back to reset the pedals after each trial. Once a mouse exceeded 70 correct trials within $20 \mathrm{~min}$, it advanced to be trained to sequentially press the assigned pedal in a fixed-ratio 2 (FR2). In this stage of the training, the step motor rotates to lock the pedals after the mice press the assigned pedal twice. Animals were initially rewarded for pressing the pedal twice between 150 and $1500 \mathrm{~ms}$. Water-reward was not given when the mice press the pedal twice within $150 \mathrm{~ms}$ (swift) or only once (over 1500 ms; overT; Fig. 4G). We appointed minimum interval to prevent mice from pressing the pedal twice too fast causing insufficient optogenetic effects. A session 
was conducted for $15 \mathrm{~min} / \mathrm{d}$. All trials were voluntarily started without any sensory cue.

\section{Fluorescent WFI}

WFI was performed on day 1 or 2 of the training step 3 (beginner) and on day 21 or 22 (expert) using a CMOS camera (FLASH4; C-13 440-20CU; Hamamatsu) system, adapted from a previous study (Kim et al., 2016). The cortical surface was imaged through a $2 \times / 0.08 \mathrm{NA}$ objective (PlanApo N; Olympus). For measurement of GCaMP6 emission, a $470 \mathrm{~nm}$ LED (Optogenetics-LED-Blue; Prizmatrix) was connected to the dichroic cube holder using a $1000-\mu \mathrm{m}$-diameter 0.50-NA fiber (M59L01; Thorlabs). The light was filtered with a $470 \mathrm{~nm}$ bandpass filter (FB470-10; Thorlabs) that was fiber-coupled into the dichroic mirror (FF495-Di03-25 $\times 36$; Semrock). Fluorescence emitted from the cortical surface was passed through a $530 \mathrm{~nm}$ bandpass fluorescence emission filter (FF01-520/35-25; Semrock). Images were acquired using $\mathrm{HClmage}$ Live (Hamamatsu) at $20 \mathrm{~Hz}$ (50 ms/frame) and $192 \times 192$ pixels. Imaging was started by using the transistor-transistor logic (TTL) signal generated from the Arduino to synchronize the imaging and behavior data.

\section{In vivo single-unit recording}

Eectrodes were custom made with four-fold twisted polyimide-coated NiCr wires (PF002005; Kanthal Precision Technology), and each wire was assembled into an electrode interface board (EIB-16 board; Neuralynx) with gold pins (Neuralynx). The electrode-interface boards were assembled into a custom-designed holder. The impedance of each tetrode channel was reduced by electro-coating with colloidal gold using a Nano-Z kit (Neuralynx). The impedance of each recording channel was $300-400 \mathrm{k} \Omega$.

At 2-3 d before the recording, mice were anesthetized with isoflurane (1.5\% in oxygen) and head-fixed with the task device. A hole was drilled in the skull above the CFA, filled with artificial CSF (ACSF), covered with a round glass (CS-3R, catalog \#64-0720, Warner Instruments), and sealed with Kwik-Sil (World Precision Instruments). On the recording day, the silicon and cover glass were removed, and the electrodes were put on Dil or $\mathrm{DiO}$ (Invitrogen) and inserted into the hole. Once a neural signal was successfully detected, the hole was sealed with $1.5 \%$ liquid agar to prevent movement from causing the signal to be lost during the task.

All mouse behaviors were recorded using an actioncam (HDR-AS200V; Sony) at $240 \mathrm{fps}$ for detailed analysis of forelimb movements. The 1-2P period for correct trials was set to $1.5 \mathrm{~s}$. Neural signals were recorded with the Cheetah 32-channel acquisition system (Neuralynx) with bandpass filtering at $3-5 \mathrm{kHz}$. The pedal-press signals obtained from the Arduino board were interconnected with the Cheetah acquisition system as TTL signals for time synchronization.

\section{Fiber photometry}

A single-wavelength time-correlated single photon counting (TCSPC) photometry system (Becker \& Hickl
$\mathrm{GmbH}$ ) was used for in vivo axon fiber calcium imaging during the biPress motor task. A $488 \mathrm{~nm}$ laser (BDL-488$\mathrm{SMC}$; Becker \& Hickl $\mathrm{GmbH}$ ) was used to excite GCaMP signals at $20 \mathrm{MHz}$ through a single-mode fiber (F100-MMFC-FET; Becker \& Hickl GmbH) connected to a multimode probe (F05-MM-FP-OPTH; Becker \& Hickl GmbH). The power measured at the free end of the optic cannula was $\sim 0.1 \mathrm{~mW}$. Photons emitted from the tissue traversed the multimode fiber before being collected by a photodetector (PMC-100; Becker \& Hickl GmbH) controlled by a detector controller (DCC-100; Becker \& Hickl GmbH), and the number of photons was counted at $20 \mathrm{~Hz}$. All mouse behaviors were recorded using action-cams at $240 \mathrm{fps}$. The Arduino board of the task device and the photometry recording system were interconnected for time synchronization. The 1-2P period cutoff for a correct trial was set to $1.5 \mathrm{~s}$.

\section{Optogenetic behavioral tests}

Patch cords (MFP_200/230/900-0.48_1m; Doric Lenses) were connected to the optic cannula and a $589 \mathrm{~nm}$ laser (MGL-F-589-100mW; Opto Engine LLC). The laser power was set to $\sim 10 \mathrm{~mW}$ as measured at the tip of the optic cannula using a power sensor (PM100D-S130VC; Thorlab). The light illumination was controlled by the Arduino program. For optogenetic manipulation during the 1-2P period, the laser was turned on right after first press $(1 \mathrm{P})$ and turned off right after second press (2P) or at $1.5 \mathrm{~s}$ (fixed interval). For optogenetic manipulation during the pre-1P period, the laser was turned on when the retracted pedals were reset and turned off right after the 1P-sided and/or 2P-sided pedals were pressed.

\section{Data analysis}

\section{WFI data analysis}

Imaging data analysis was performed with customized MATLAB codes (MathWorks). To align brain images from different sessions, a two-dimensional cross-correlation between images was calculated to estimate the amount of spatial shift that should be applied to each image. Images from different mice were aligned using the position of the bregma. To reduce any background noise, each brain image was smoothed with a two-dimensional Gaussian filter ( $\sigma=2$ pixels).

The rostral forelimb area (RFA) and CFA regions were determined based on motor maps generated by previous intracortical microstimulation experiments (Tennant et al., 2011; Hira et al., 2013). The coordinates were AP +1.52.7; $\mathrm{ML} \pm 0.75-1.75 \mathrm{~mm}$ for the RFA and AP +0.8 to -0.8 ; $\mathrm{ML} \pm 1.2-2.5 \mathrm{~mm}$ for the CFA. The region of visual area (VIS) was determined based on the Allen Brain Atlas and the coordinates were AP -2.59-5.17; ML $\pm 1.23-3.87 \mathrm{~mm}$.

Time series data on the activity in each pixel was filtered between 0.1 and $9.9 \mathrm{~Hz}$. Each correct trial was defined as spanning $1250 \mathrm{~ms}$ before $1 \mathrm{P}$ to $1250 \mathrm{~ms}$ after $2 \mathrm{P}$. For incorrect trials, the trial end was defined as $1250 \mathrm{~ms}$ after the time cutoff for an overT trial or $2000 \mathrm{~ms}$ after $1 \mathrm{P}$ for bothP and wrongP trials. Samples which data lay beyond $3 \sigma$ (SD) of the entire dataset were excluded from the analysis. 
To obtain $\Delta F / F$ values for each pixel, the baseline fluorescence $(F)$ was estimated from the average activity of the non-sample periods. The series average of $\Delta F / F$ was smoothed over time with a Gaussian filter ( $\sigma=150 \mathrm{~ms}$ ). To detect a behavior-related peak, the peak of activity was searched within a $-500-$ to $+500-\mathrm{ms}$ window of each behavioral press. Since the calcium signals were weak and showed multiple peaks in the VIS, samples were smoothed with a Gaussian filter $(\sigma=500 \mathrm{~ms})$ and those showing peak values larger than mean + SD of sample activity were included in the analysis to find a significant peak activity.

\section{Behavior video analysis for in vivo single-unit and GCaMP photometry}

The movement start (MovS) of each event was determined as the time when the animal started to move its forelimb after the rest period. The $1 \mathrm{P}$ and $2 \mathrm{P}$ events were indicated by red and green LEDs signaled from the Arduino; thus, the interval between the MovS and $1 \mathrm{P}$ events could be calculated through frame-by-frame video analysis. We did not specifically classify movement types for the MovS in this study. It included either movements of 1P-related or 2P-related forelimb. A rest period was defined as a period during when the mouse showed no movement for $>1 \mathrm{~s}$. Trials without a rest period were excluded from the data analysis.

In vivo single-unit recording

For single-unit isolation, spikes were extracted and clustered using the Spike Extractor and SpikeSort 3D software packages (Neuralynx). Only high-quality isolated units, which were defined as having Lratio $<0.1$ and isolation distance >15 (Kim et al., 2017; Lee et al., 2012) were selected for data analysis.

Samples shorter than $5 \mathrm{~s}$ were selected for analysis. The instantaneous firing rate was calculated by applying a Gaussian filter $(\sigma=100 \mathrm{~ms})$ to the observed spike activities. For quantitative comparison of firing patterns, the instantaneous firing rate during the various intervals [i.e., MovS-1P, 1-2P (for a correct trial) or 1P-trial end (for an incorrect trial)] were linearly interpolated to match the activity length by rescaling to $1 \mathrm{~s}$ for MovS-1P, to $0.5 \mathrm{~s}$ for $1-$ $2 \mathrm{P}$ or to $0.6 \mathrm{~s}$ for $1 \mathrm{P}$-trial end.

For classification of neural firing patterns, a time window of $100 \mathrm{~ms}$ before and after the press timing was considered. The firing rate in each window was z-scored within each neuron. We classified activities for each behavioral event (MovS, 1P, and 2P) into two groups using k-means clustering method because the Silhouette index was maximized for the two groups $(k=2-6)$. Neurons could be classified into eight groups according to the combination of behavior patterns (Extended Data Fig. 3$1 B, C, n=171)$.

Principal component analysis of average neural firing rates was performed using MATLAB (MathWorks). To find neurons that encoded correct and incorrect trials differently, the cumulative curves of differences in the firing patterns ( $\Delta$ Firing Rate, $\Delta \mathrm{FR}$ ) between correct and incorrect trials were classified using $\mathrm{k}$-means clustering into $k$ groups $(k=2-6)$. We then measured the Silhouette index across the clusters to determine the optimal number of clusters, which was two groups. Using the observed neural activities of each group, we have decoded whether each trial was correct or incorrect. A trial performance decoder (determining whether the trial was correct or incorrect) was implemented (fivefold, 200-ms window, 100-ms shift, 50 repetitions) using the support vector machine (SVM) algorithm.

\section{Fiber photometry data analysis}

All procedures were performed as described for the single-unit data analysis. The total photon count was obtained over $50 \mathrm{~ms}$, and the time series of the fluorescent signals was filtered between 0.1 and $9.9 \mathrm{~Hz}$. To obtain $\Delta F /$ $F$ values, the baseline fluorescent signal $(F)$ was estimated from the trial start (Trial-S)-MovS period of each trial and filtered in time with a Gaussian filter ( $\sigma=150 \mathrm{~ms})$.

\section{Histology}

Mice were deeply anesthetized with 2,2,2-tribromoethanol $(20 \mathrm{mg} / \mathrm{ml}$, i.p.; Sigma T48402) or a mixture of ketamine $(100 \mathrm{mg} / \mathrm{kg})$ and dexmedetomidine $(0.5 \mathrm{mg} / \mathrm{kg})$. The animals were then perfused and fixed with the $0.9 \%$ saline and $4 \%$ formaldehyde in PBS. Brains were removed, postfixed in $4 \%$ formaldehyde overnight at $4{ }^{\circ} \mathrm{C}$, sectioned into 50- $\mu \mathrm{m}$ coronal slices using a vibratome (VT1200S; Leica) and mounted onto glass slides with DAPI mounting medium solution ( $\mathrm{H}-1500$; Vector Labs). Images were taken under a fluorescent microscope (VS120; Olympus) or a confocal microscope (KAIST Biocore center, LSM780; Zeiss).

\section{Statistics}

Statistical analyses were performed using SigmaPlot12 (Systat Software) and MATLAB (MathWorks). For WFI analysis, we used Wilcoxon signed-rank test, two-tailed paired $t$ test and one-way repeated-measures (RM) ANOVA with Tukey-Kramer multiple comparisons test. For singleunit data analysis, we applied Wilcoxon signed-rank test, Friedman test, two-sample $t$ test. For fiber photometry data analysis, we used Wilcoxon signed-rank test. For optogenetic experiments, we used two-tailed paired/unpaired $t$ tests, and the nonparametric Wilcoxon signed-rank test and Mann-Whitney $U$ test. Normality and equal variance of the data distribution were examined; $p<0.05$ was considered statistically significant.

\section{Results}

\section{Coherent neuronal activities in the two motor cortical hemispheres during the biPress sequence task}

We first designed a biPress sequence task in which head-fixed mice were trained only to press the right (1P) and then left $(2 \mathrm{P})$ pedals, sequentially, or vice versa (Fig. 1A; Extended Data Fig. 1-1; Movies 1, 2, 3, 4). The mice were initially water-rewarded when they completed $2 \mathrm{P}$ within $5 \mathrm{~s}$ after $1 \mathrm{P}$. The allowed time interval between $1 \mathrm{P}$ and $2 \mathrm{P}$ (the 1-2P period) was shortened by $0.5 \mathrm{~s}$ for every 10 correct trials, down to a minimal duration of $1.5 \mathrm{~s}$ (Fig. $1 A)$. The forelimb for $1 \mathrm{P}$ was positioned to the pedal and the other forelimb was placed into the floor to be balanced. These positions were switched each other for $2 \mathrm{P}$ 


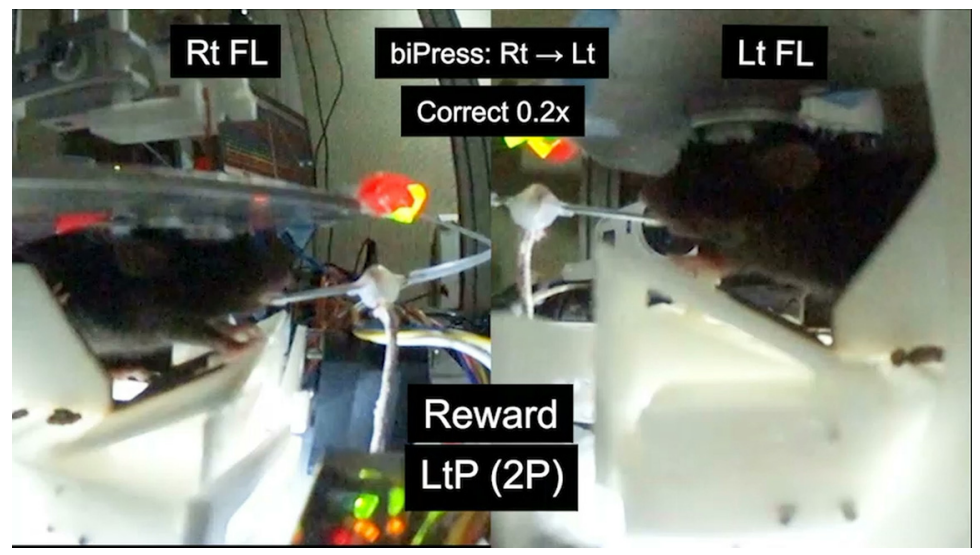

Movie 1. Example movie of a mouse performing the biPress task. Movie shows representative correct trial in the same mouse with the view of each forelimb. The movie is played $1 \times$ speed and then $0.2 \times$ slow motion. The mouse was trained to press the pedals in a right $\rightarrow$ left sequence. The $1 \mathrm{P}, 2 \mathrm{P}$, and correct trial were indicated by the red, green, and yellow LED, respectively. These LEDs were embedded only during the recording day for analysis. [View online]

(Movie 1). Incorrect trials included instances of mice not pressing $2 \mathrm{P}$ within the desired time interval (overT), pressing both pedals together (bothP), and pressing the wrong side as 1P (wrongP; Fig. 1A; Movies 2, 3, 4). Although the mice showed similar movement patterns for $1 \mathrm{P}$ but did not show any movements after $1 \mathrm{P}$ causing overT incorrect trials (Movie 2). Meanwhile, in bothP trials, the mice positioned both forelimbs to each pedal and pressed them almost simultaneously (Movie 3). In wrongP incorrect trials, the 1P-related forelimb was moved but not placed it into the pedal, and then the other forelimb was performed the pedal-press (Movie 4). After three weeks of training, the mice reduced unnecessary movements and showed a higher ratio of correct trials than incorrect trials and decreases in the length and variability of the 1-2P period compared with those observed during the beginning phases of the training ( $n=6$ mice, left, one-way RM ANOVA with Tukey's post hoc test, $F_{(22,137)}=14.953$, $p<0.001$, right, one-way RM ANOVA with Tukey's post hoc test, $\chi^{2}=62.094$ with 22 degrees of freedom, $p<0.001$; Fig. $1 B$ ). As the mice learned to sequentially press the pedals during the training, the ratio of overT to total incorrect trials also increased compared with that of the other incorrect trial types ( $n=6$ mice, left, two-way RM ANOVA, $F_{(22,413)}=3.839, p<0.001$ for days, $F_{(44,413)}=$ $1.905, p=0.001$ for days $\times$ type, right, two-tailed paired $t$ test, orange, $p=0.0273$ for day 1 vs day 3 , Wilcoxon signed-rank test, green, $p=0.031$ for day 1 vs day 2; twotailed paired $t$ test, green, $p=0.0185$ for day 1 vs day 3 ; Fig. $1 C$ ), suggesting that the mice successfully learned the biPress motor task.

Next, to investigate the cortical dynamics of the hemispheres, we used Thy1-GCaMP6s mice harboring the genetically encoded $\mathrm{Ca}^{2+}$ indicator, GCaMP6s, in their cortical neurons (Wekselblatt et al., 2016; Makino et al., 2017) and performed wide-field $\mathrm{Ca}^{2+}$ imaging (WFI) on the left and right cortices during the biPress task training (Fig. 2). The 1P-related and $2 \mathrm{P}$-related $\mathrm{Ca}^{2+}$ signals increased mainly in the cortical areas known as the RFA and the CFA (Tennant et al., 2011; Hira et al., 2013) during

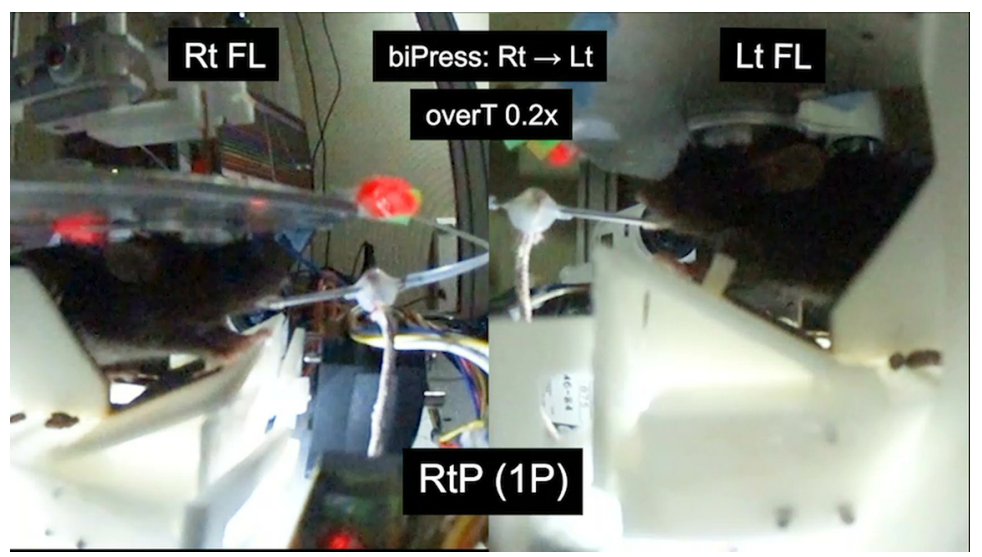

Movie 2. Example movie of a mouse performing the biPress task. Movie shows representative overT trial in the same mouse with the view of each forelimb. The movie is played $1 \times$ speed and then $0.2 \times$ slow motion. The mouse was trained to press the pedals in a right $\rightarrow$ left sequence. The $1 \mathrm{P}, 2 \mathrm{P}$, and correct trial were indicated by the red, green, and yellow LED, respectively. These LEDs were embedded only during the recording day for analysis. [View online] 


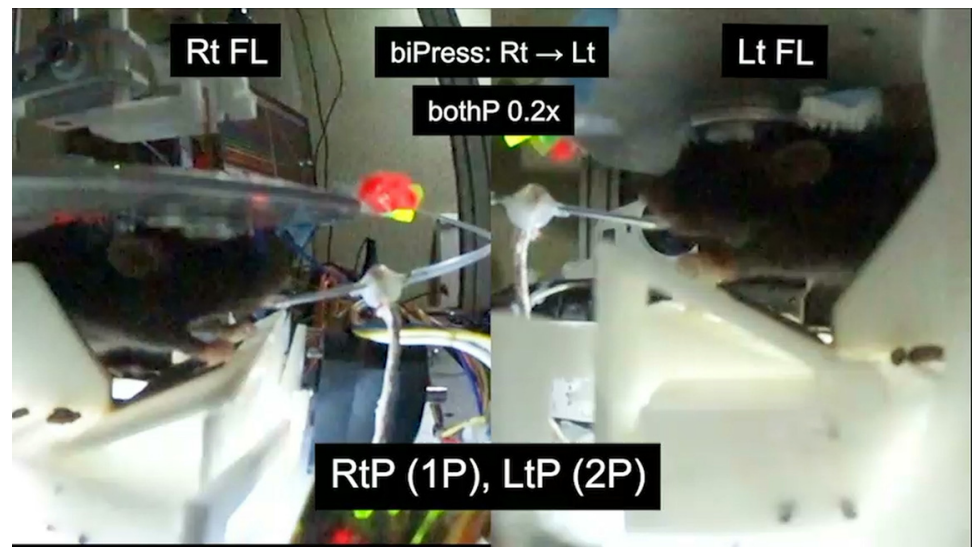

Movie 3. Example movie of a mouse performing the biPress task. Movie shows representative bothP trial in the same mouse with the view of each forelimb. The movie is played $1 \times$ speed and then $0.2 \times$ slow motion. The mouse was trained to press the pedals in a right $\rightarrow$ left sequence. The $1 \mathrm{P}, 2 \mathrm{P}$, and correct trial were indicated by the red, green, and yellow LED, respectively. These LEDs were embedded only during the recording day for analysis. [View online]

the task (Fig. 2A-C). Moreover, the CFA showed higher $\triangle F / F$ signals than the RFA (Fig. $2 C$, left, one-way RM ANOVA with Tukey's post hoc test, $F_{(2,12)}=37.35$, $p=7.03 \times 10^{-6}$ for the $1 \mathrm{P}$-related hemisphere, $F_{(2,12)}=$ 14.1, $p=7.10 \times 10^{-4}$ for the $2 \mathrm{P}$-related hemisphere; Fig. $2 C$, right, one-way RM ANOVA with Tukey's post hoc test, $F_{(2,12)}=52.28, p=1.19 \times 10^{-6}$ for the 1 P-related hemisphere, $F_{(2,12)}=23.80, p=6.66 \times 10^{-5}$ for the $2 \mathrm{P}$ related hemisphere, Two-tailed paired $t$ test, $p=0.021$ for 1P-CFA vs 2P-CFA, $p=0.23$ for 1P-RFA vs 2P-RFA, $p=0.062$ for $1 \mathrm{P}-\mathrm{VIS}$ vs $2 \mathrm{P}-\mathrm{VIS})$. We also found that two hemispheres represented each corresponding forelimb movements. Compared with the 1P-related hemisphere, the $\mathrm{Ca}^{2+}$ signals level of the 2P-related hemisphere was lower in correct and overT incorrect trials but higher in wrongP incorrect trials during the 1P timing. In case of bothP incorrect trials, involved simultaneous movements of both forelimbs, there was no significant difference between the two hemispheres $\left(p=6.85 \times 10^{-4}\right.$ for correct, $p=2.73 \times 10^{-5}$ for overT, $p=0.367$ for bothP, $p=0.0072$ for wrongP, two-tailed paired $t$ test, Jackknife resampling; Extended Data Fig. 2-1A)

Interestingly, the sequential peaks of $1 \mathrm{P}$-related and 2P-related cortical activities of both the CFA and RFA was observed in correct trials but not in incorrect trials during the task. This peak interval between $1 \mathrm{P}$-related and 2Prelated motor cortical areas was shortened and became less variable at week 3 of training (experts) compared with the those in the beginning of training (beginners), which was consistent with our behavioral patterns [Fig. $2 D-L$; two-tailed paired $t$ test, $p=0.41$ for 1P-CFA, $p=4.67 \times$ $10^{-4}$ for 2P-CFA (Fig. 2D, left) and two-tailed paired $t$ test, $p=0.26$ for 1 P-RFA, $p=3.35 \times 10^{-4}$ for 2P-RFA (Fig. $2 D$, right); 1P-related hemisphere: $0.0913 \mathrm{~s}$ vs $2 \mathrm{P}$-related hemisphere: $1.11 \mathrm{~s}, p=0.0156$ (Fig. 2F); 1P-related hemisphere: $0.134 \mathrm{~s}$ vs 2P-related hemisphere: $0.284 \mathrm{~s}$, $p=0.0156$ (Fig. 2H); 1P-related hemisphere: $0.135 \mathrm{~s}$ vs 2P-related hemisphere: $1.21 \mathrm{~s}, p=0.0156$ (Fig. 2J); 1P-related hemisphere: $0.145 \mathrm{~s}$ vs $2 \mathrm{P}$-related hemisphere: $0.276 \mathrm{~s}, p=0.0313$, mice $n=7$, Wilcoxon signed-rank test (Fig. 2L)]. Although the $\mathrm{Ca}^{2+}$ signals in the $2 \mathrm{P}$-related

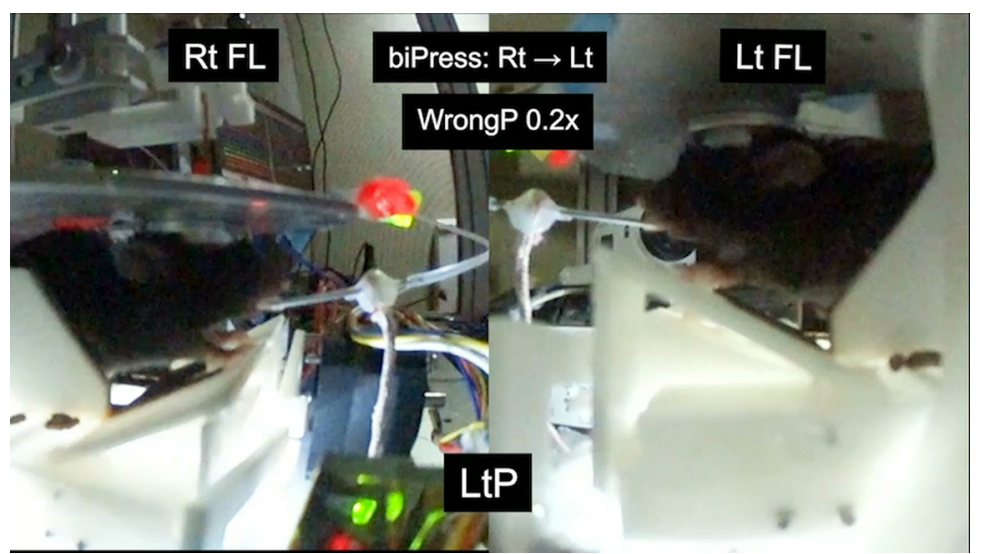

Movie 4. Example movie of a mouse performing the biPress task. Movie shows representative wrongP trial in the same mouse with the view of each forelimb. The movie is played $1 \times$ speed and then $0.2 \times$ slow motion. The mouse was trained to press the pedals in a right $\rightarrow$ left sequence. The $1 \mathrm{P}, 2 \mathrm{P}$, and correct trial were indicated by the red, green, and yellow LED, respectively. These LEDs were embedded only during the recording day for analysis. [View online] 
A

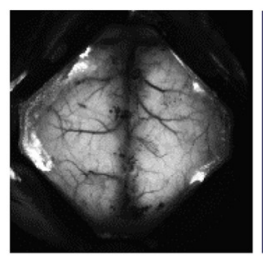

B

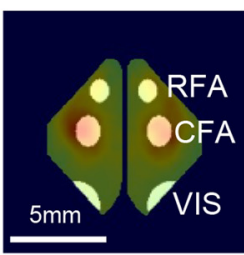

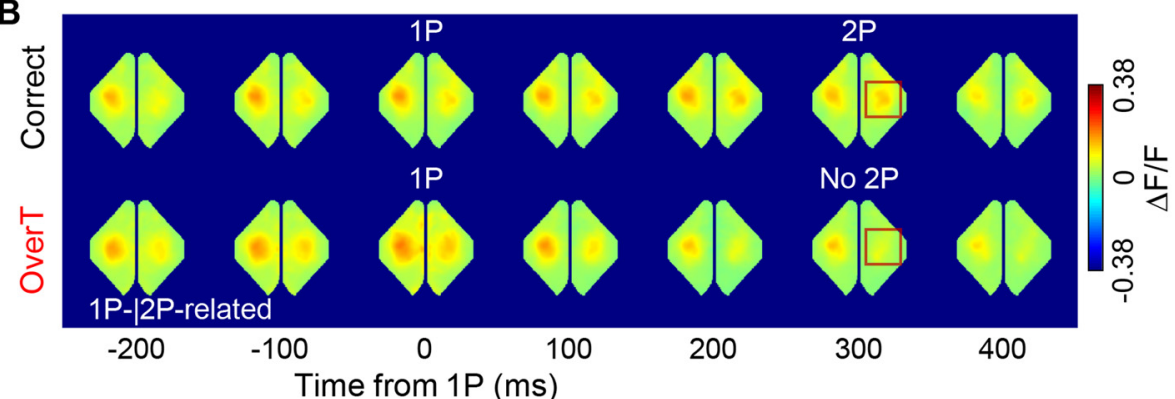

C
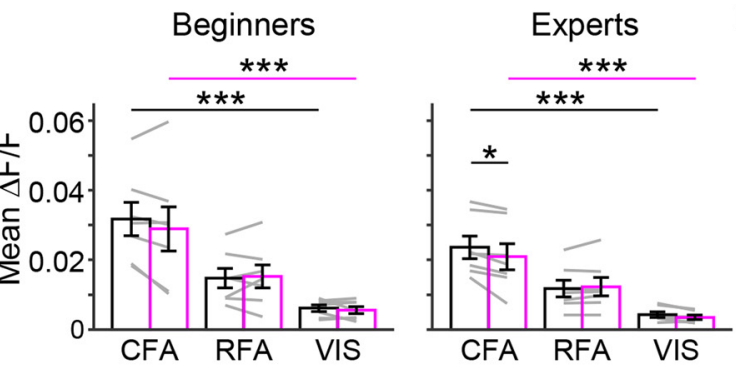

D

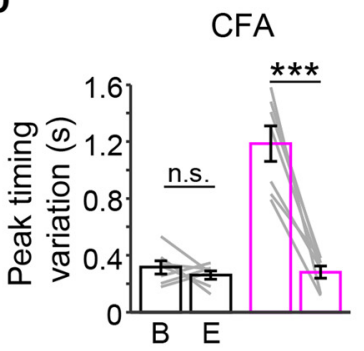

RFA
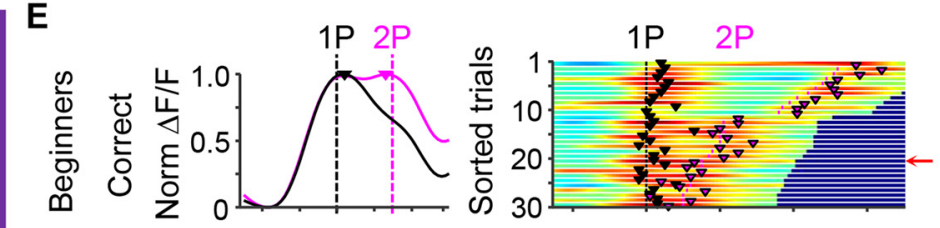

$\mathbf{F}$

G
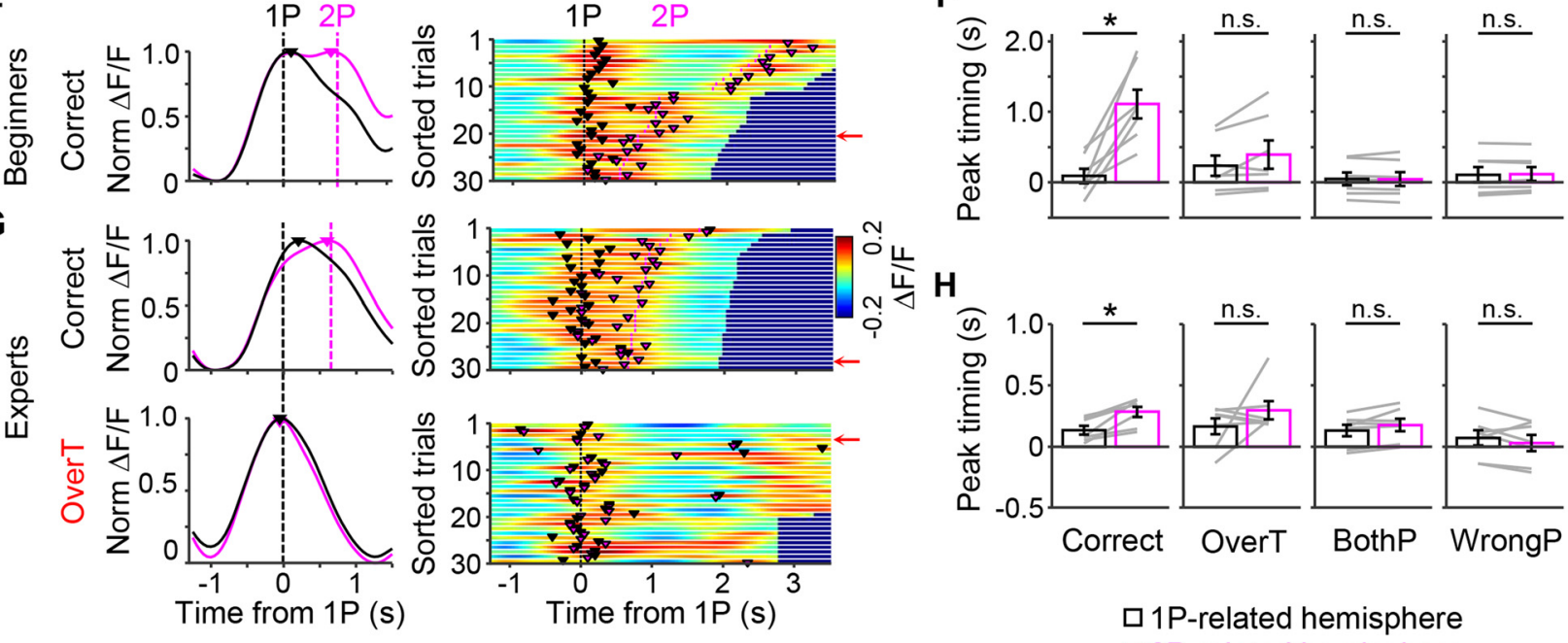

$\stackrel{\mathbb{U}}{\mathrm{U}}$
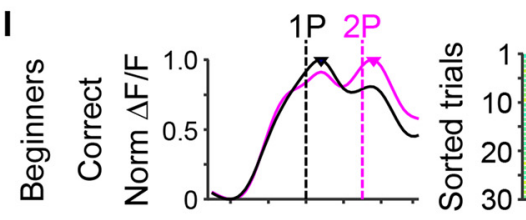

$1 \mathrm{P} \quad 2 \mathrm{P}$

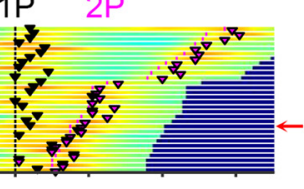

K
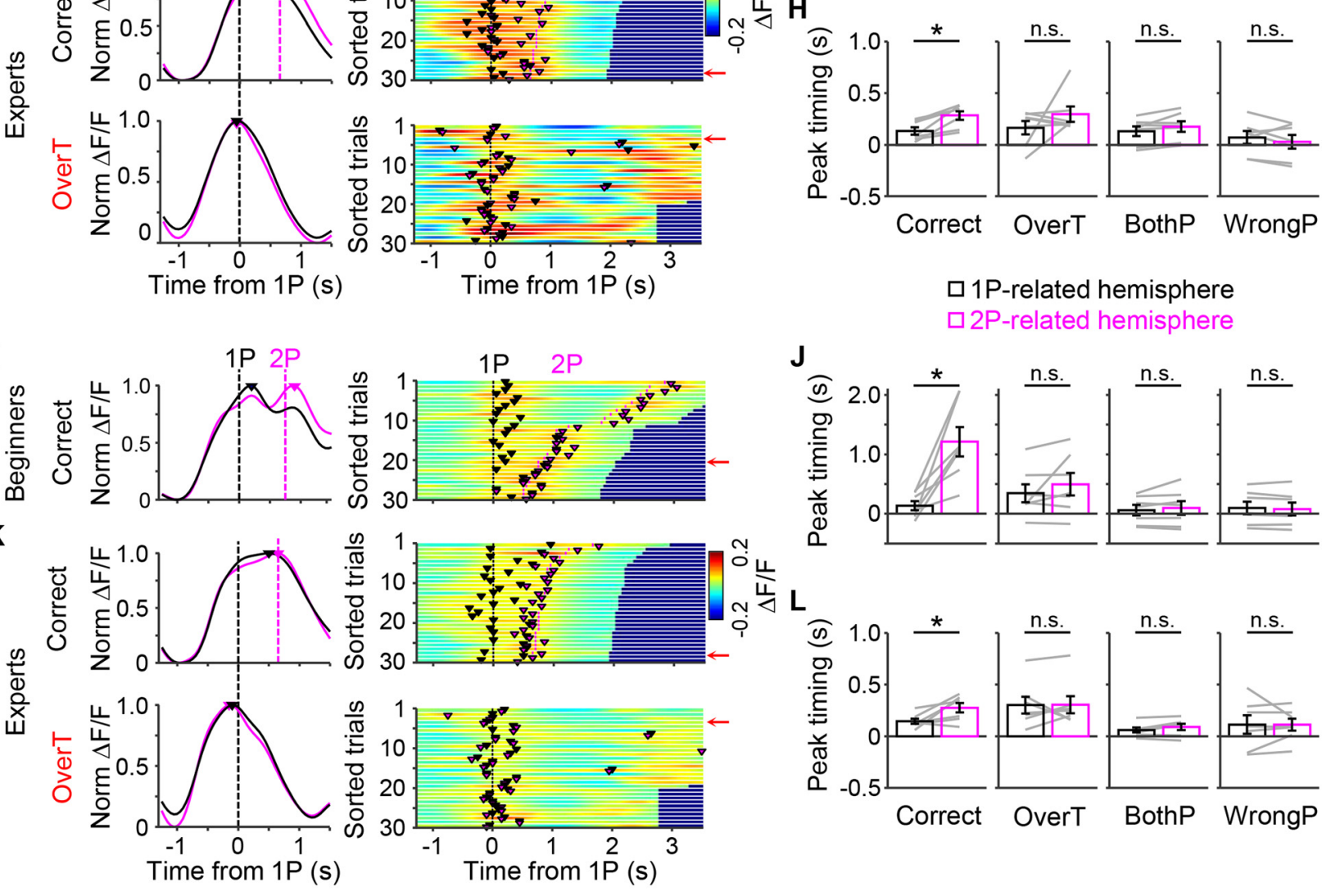

$\square$ 1P-related hemisphere

$\square 2 \mathrm{P}$-related hemisphere

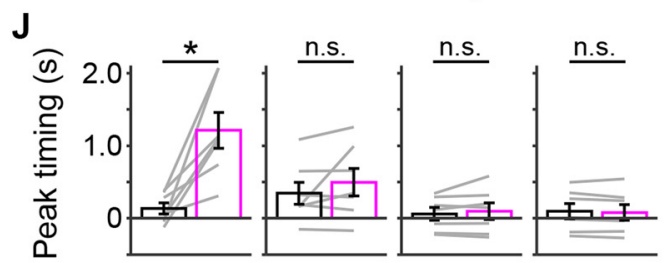

$\underset{\mathbb{1}}{\mathbb{x}}$

Figure 2. Sequential $\mathrm{Ca}^{2+}$ activities between the two motor cortical areas during the biPress sequence task in head-fixed mice. $\boldsymbol{A}$, left, A field of view of wide-field $\mathrm{Ca}^{2+}$ imaging in the mouse cortex. Right, Motor area (CFA, RFA) and VIS were examined. $\boldsymbol{B}$, 
continued

Sample patterns of cortical activity represented as changes in GCaMP6 signals during the biPress task. Red squares indicate differences between correct and incorrect trials. $\boldsymbol{C}$, The average $\triangle F / F$ in the CFA, RFA, and VIS. $\boldsymbol{D}$, Peak timing variation across the brain regions. Left, Beginners versus experts in the CFA. Right, Beginners versus experts in the RFA. B, beginners; E, experts. $\boldsymbol{E}$, G, Representative activity patterns in the CFA of beginner $(\boldsymbol{E})$ and expert $(\boldsymbol{G})$ mice. Left, Sample activity pattern in a single trial. Black and magenta curves represent normalized $\Delta \mathrm{F} / \mathrm{F}$ activities of the $1 \mathrm{P}$-related and $2 \mathrm{P}$-related hemispheres, respectively. Vertical dashed lines indicate the time points of $1 \mathrm{P}$ (black) and 2P (magenta). Colored triangles represent the activity peak timing. Right, Population activities shown as a heatmap. Colored triangles and vertical lines represent the activity peak timing and the timing of $1 \mathrm{P}$ (black) and 2P (magenta), respectively. Trials were sorted by the duration. Red arrows indicate the sample trials presented in the left panel. $\boldsymbol{F}, \boldsymbol{H}$, Comparison between the peak timings of 1P-CFA and 2P-CFA of the beginner $(\boldsymbol{F})$ and expert $(\boldsymbol{H})$ mice. Black and magenta boxes represent the averaged activity peak timings in the 1P-related and 2P-related hemispheres, respectively. Gray lines represent individual data. $\boldsymbol{I}, \boldsymbol{K}$, Representative activity patterns in the RFA of beginner $(\boldsymbol{I})$ and expert $(\boldsymbol{K})$ mice. $\boldsymbol{J}, \boldsymbol{L}$, Comparison between the peak timings of 1P-RFA and 2P-RFA of the beginner $(\boldsymbol{J})$ and expert $(\boldsymbol{L})$ mice. Same format as $\boldsymbol{E}-\boldsymbol{H}$. All error bars represent SEM; ${ }^{\star} p<0.05,{ }^{\star \star \star} p<0.001$, n.s. not significant Comparisons of $\Delta \mathrm{F} / \mathrm{F}$ between the $1 \mathrm{P}$-related and $2 \mathrm{P}$-related hemispheres were shown in Extended Data Figure 2-1A,B. Comparisons of peak timings in the VIS were shown in Extended Data Figure 2-1C.

Figure Contributions: Minju Jeong performed the experiments. Hyeonsu Lee analyzed the data.

hemisphere exhibited arisen during the $1 \mathrm{P}$ even before $2 \mathrm{P}$ was performed, the signals level was weaker during the $2 \mathrm{P}$ than the $1 \mathrm{P}$ timing in the correct trials $\left(p=2.80 \times 10^{-5}\right.$, two-tailed paired $t$ test, Jackknife resampling; Extended Data Fig. 2-1B). We also confirmed that this sequential activity peak in the correct trials was not observed between left and right hemispheres of the VIS ( $n=7$ mice, Wilcoxon signed-rank test, $p>0.05$; Extended Data Fig. 2-1C).

\section{The motor cortex responsible for the 1P also generates motor signals for the $2 \mathrm{P}$ by ipsilateral forelimb}

The WFI data led us to consider how the activity sequence is organized between the motor cortices. The results suggested that information flow for successful task performance would occur from $1 \mathrm{P}$-motor area (contralateral to the $1 \mathrm{P}$ forelimb) to $2 \mathrm{P}$-motor area (contralateral to the 2P forelimb; Fig. 2E-L; Extended Data Fig. 2-1B). Therefore, we hypothesized that the 1P-motor area might also encode information about the subsequent $2 \mathrm{P}$ by the opposite forelimb and transfer these motor signals before the $2 \mathrm{P}$ movements are actually generated. In particular, there might exist neurons whose firing rates increase during the period between after $1 \mathrm{P}$ and before $2 \mathrm{P}$ (1-2P period), and these neural signals would play important roles in continuous $2 \mathrm{P}$ performance of the biPress sequence task.

To identify the neural signals for correct $2 \mathrm{P}$ movements in the sequential biPress task, we performed extracellular single-unit recordings in the $1 \mathrm{P}-\mathrm{CFA}$ of fully trained mice (Fig. 3A; Extended Data Fig. 3-1A). Then, we examined and classified firing patterns for each behavioral events as follows: $1 \mathrm{~s}$ before the start of movement (MovS; Trial-S), MovS, 1P, 2P, and $0.1 \mathrm{~s}$ after 2P (Trial-E; Fig. $3 A$ ). Since we found that bothP and wrongP incorrect trials were caused by mistaken $1 \mathrm{P}$, we further investigated differences between correct and overT incorrect trials. Most of the 1P-CFA neurons increased their firing rates around the MovS and/or $1 \mathrm{P}$ events $(82.5 \%, n=141 / 171)$, and there also existed the 1P-CFA neurons which firing rate increased around $2 \mathrm{P}$ event $(37.4 \%, n=64 / 171)$. Interestingly, these $2 \mathrm{P}$-related neurons showed no such increase during overT trials (Extended Data Fig. 3-1B, blue dashed boxes). Therefore, to confirm the strong correlation between these $2 \mathrm{P}$-related activities and correct $2 \mathrm{P}$ performance, we scrutinized firing pattern differences between correct and over $T$ incorrect trials.

Comparison of activity patterns between correct and overT incorrect trials also showed significant differences during the 1-2P period (Fig. $3 B-D$ ). BothP and wrong P incorrect trials also showed lower activity than correct trials during the 1-2P period. However, this activity decrease was also observed during the period between MovS and $1 \mathrm{P}$ (pre-1P) in bothP and wrongP trials [Wilcoxon signedrank test, $p<0.05$ (Fig. 3B, left); Friedman test with Tukey's post hoc test, ${ }^{*} p<0.05,{ }^{* *} p<0.01$ (Fig. 3B, right)]. In addition, to examine whether the neural activity during the 1-2P period could discriminate correct and overT incorrect task performances, we performed a classification test for neural decoding. We firstly classified the 1P-CFA neurons into two groups: those that showed differences between correct and overT incorrect trials (type1) and those that did not (type2; Fig. 3E, left). Then, we separately trained a SVM with neural activities of correct and overT incorrect trials of each group, respectively. As expected, this decoding analysis showed higher accuracy in the type1 group than the type2 group during the 1-2P period (two-sample $t$ test, $p<0.05$; Fig. $3 E$, right), indicating that the decoder could more clearly classify behavioral performance from neurons with the higher activity during the 1-2P periods. Together these results suggest that the 1P-CFA neurons encode neural signals required for a correct performance of $2 \mathrm{P}$ during the $1-2 \mathrm{P}$ period.

\section{The 2P-related motor signals from the 1P-CFA neurons are transferred to the 2P-CFA via the corticocortical pathways}

To activate the 2P-CFA neurons of the opposite hemisphere for generation of the $2 \mathrm{P}$ forelimb movement, we speculated that the 1P-CFA neurons might send signals through the corticocortical projections during those movement transition periods. To examine this possibility, we measured the axon-terminal $\mathrm{Ca}^{2+}$ signals of the $1 \mathrm{P}$ CFA neurons in the 2P-CFA by injecting an AAV-expressing GCaMP6f (AAV-GCaMP6f) into the 1P-CFA and 
A

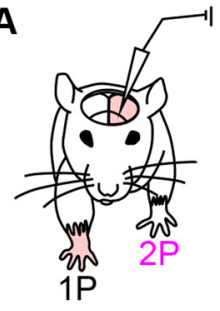

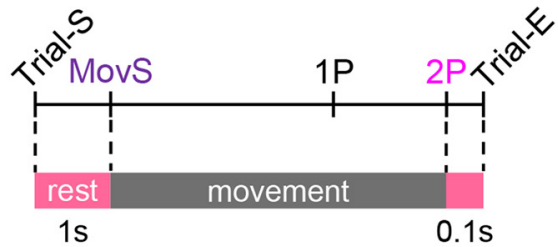

B

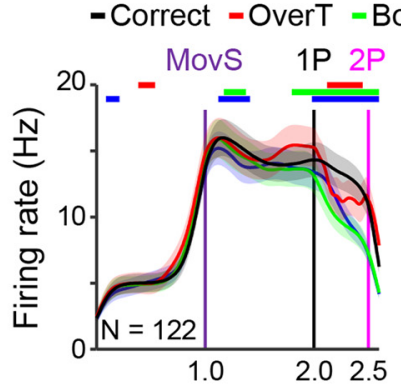

Rescaled time (A.U.)

D

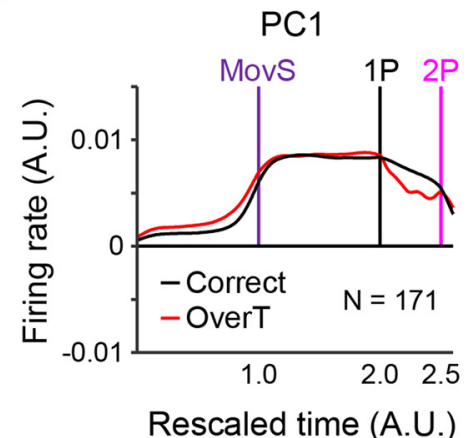

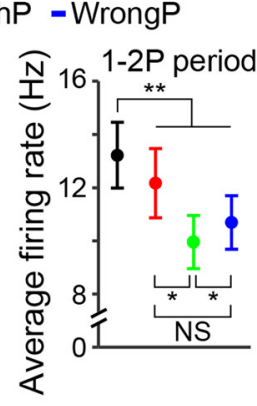

C

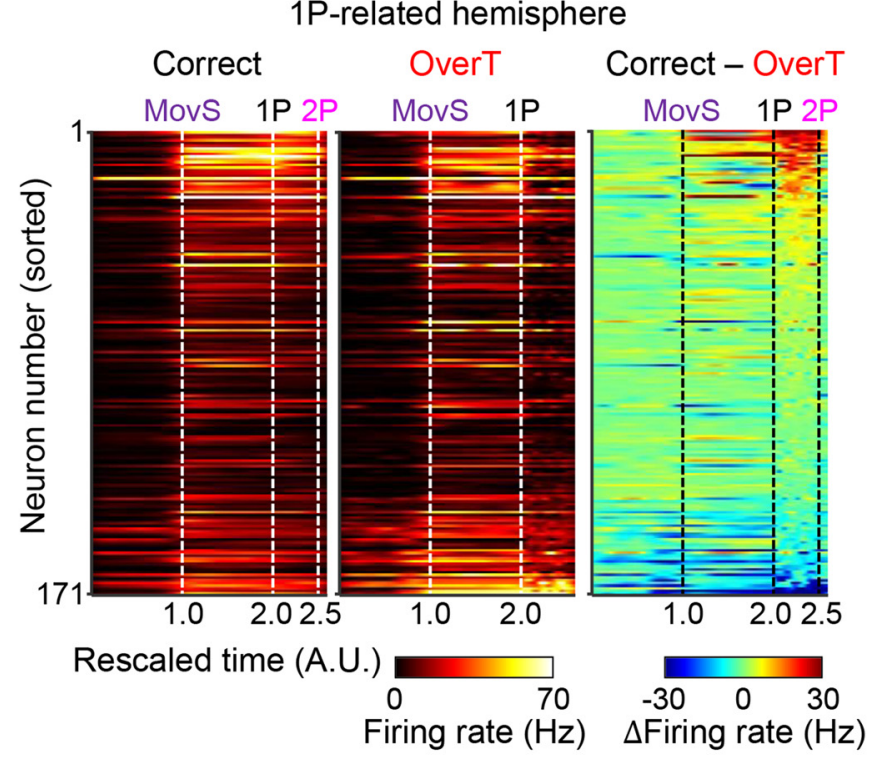

E

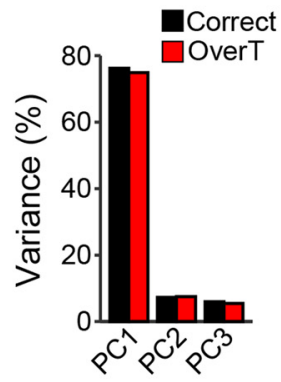

Type 1

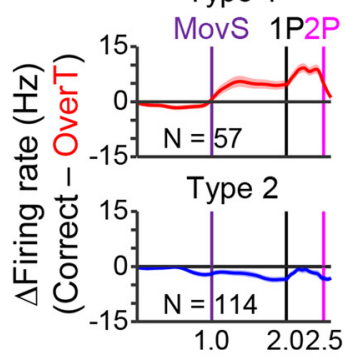

Rescaled time (A.U.)

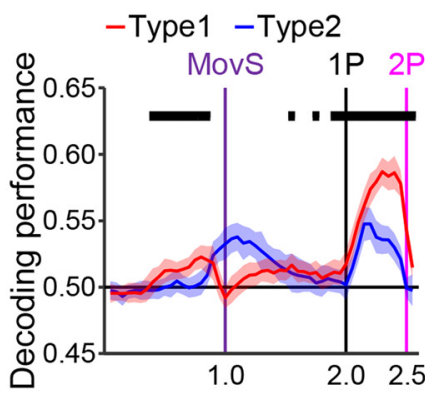

Rescaled time (A.U.)

Figure 3. Increased neural activities in the 1P-CFA during the 1-2P period involve in correct performance of the biPress task. $\boldsymbol{A}$, Schematic illustration of experimental and trial designs. For location of electrodes, see Extended Data Figure 3-1A. B, left, Averaged firing rates in correct and incorrect trials. Vertical lines represent the time points of MovS (purple), 1P (black) and 2P (magenta). Colored horizontal bars indicate periods of statistically different firing rate (correct $>$ incorrect). Firing patterns and the ratio of neural population sorted by each behavioral event were shown in Extended Data Figure 3-1B,C. Right, Averaged firing rates during the interpress interval (for averaged firing rates during the pre-1P periods, see Extended Data Fig. 3-1D). Shaded areas and error bars represent SEM. ${ }^{*} p<0.05,{ }^{* *} p<0.01$, NS not significant. $\boldsymbol{C}$, Neural activity profiles sorted by the level of firing rate difference observed during the interpress interval. $\boldsymbol{D}$, Principal component analysis (PCA) of firing patterns. Left, The first component (PC1). Right, The variance explained by each component. $\boldsymbol{E}$, Two groups of neurons were classified by their difference in activity between correct and overT incorrect trials. Left, Average activity pattern in each group. Right, Decoding performance analysis. Shaded areas represent SEM. Black horizontal bars indicate statistically different periods (type1 > type2).

Figure Contributions: Minju Jeong and Youngsoo Kim performed the experiments. Hyeonsu Lee analyzed the data.

implanting an optic cannula in the 2P-CFA (Fig. 4A). Using this system, we observed that the activity of axon terminals of $1 \mathrm{P}$-neurons in the 2P-CFA is highly elevated during 1-2P periods in correct trials (Fig. 4B), but not in overT incorrect trials [Mann-Whitney $U$ test, $p<0.05$ (Fig. 4C, left); MannWhitney $U$ test, $p=0.0088$ (Fig. $4 C$, right)], suggesting that transfer of motor signals from 1P-CFA to $2 \mathrm{P}-\mathrm{CFA}$ is important for 2P movements in the biPress task.

To determine functional contribution of neural activity hemispheric transfers during the $1-2 \mathrm{P}$ period in the biPress task, we performed the optogenetic inactivation of corticocortical projections. To selectively inhibit the neural activities of the 1P-CFA corticocortical afferents, we injected an AAV harboring genes for the Cre- recombinase-dependent expression of Archaerhodopsin (AAV-Flex-ArchT) into the 1P-CFA, a retrogradely transduced AAV expressing Cre recombinase (retroAAV-Cre) into the 2P-CFA and then implanted an optic cannula into the

2P-CFA (Fig. 4D). Photoinhibition of the 1P-CFA axon terminals in the 2P-CFA during the 1-2P period significantly reduced the ratio of correct trials caused by the increase of overT incorrect trials $(n=5$ mCherry control and $n=8$ ArchT mice, left, two-tailed paired $t$ test, $p=0.082$ for mCherry, Wilcoxon signed-rank test, $p=0.016$ for ArchT, right, Mann-Whitney $U$ test, $p=0.03$ for correct, $p=0.045$ for overT, $p=0.622$ for wrongP, two-tailed unpaired $t$ test, $p=0.947$ for bothP; Fig. $4 E$ ). In contrast, photoinhibition 
A

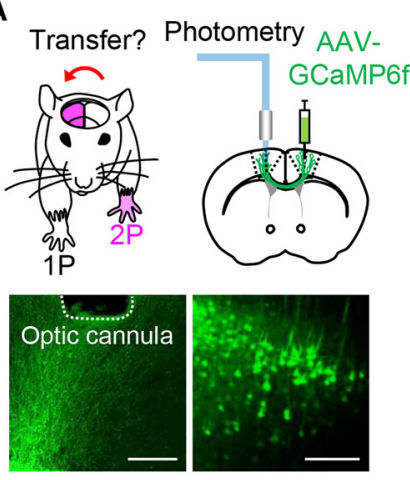

D

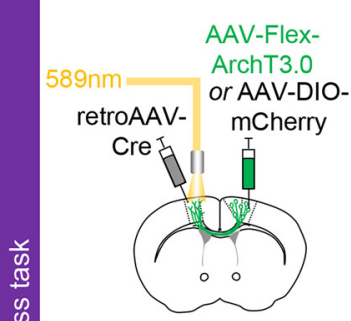

क⿺

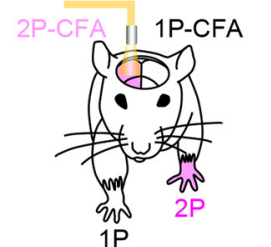

Control
B

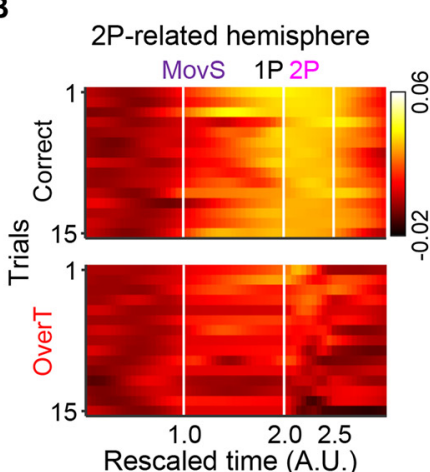

C

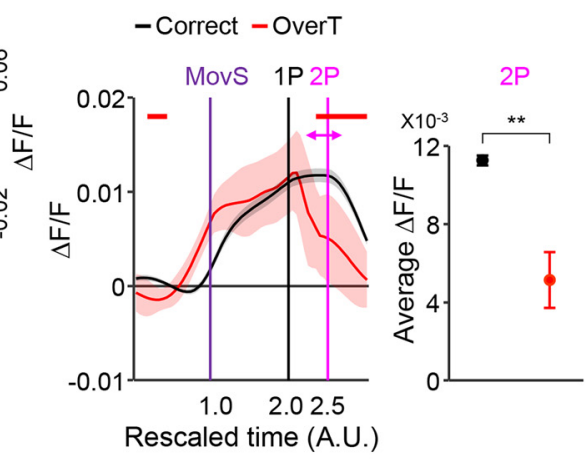

E

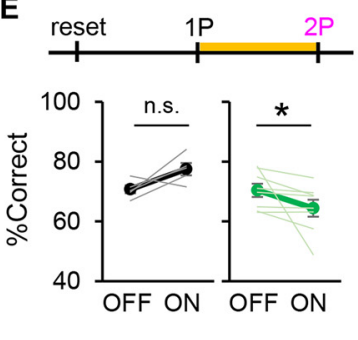

$\mathbf{F}$
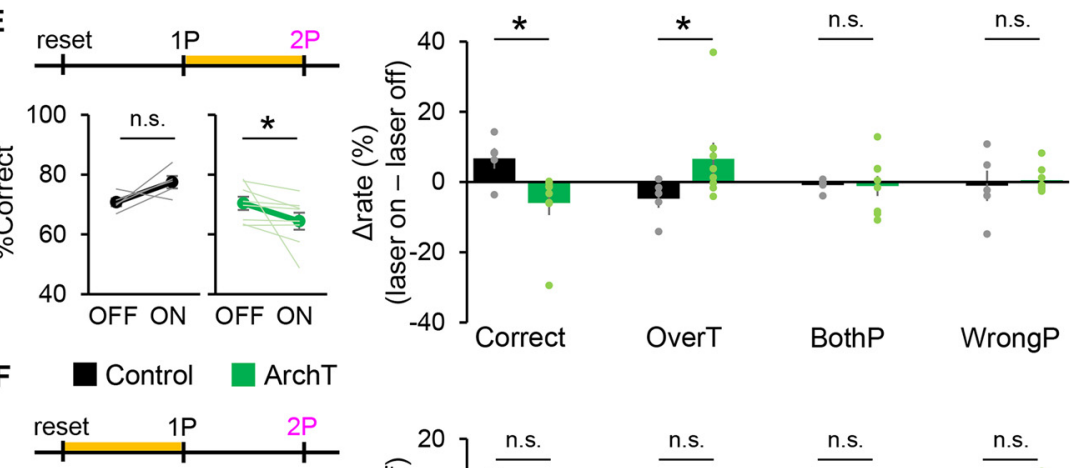
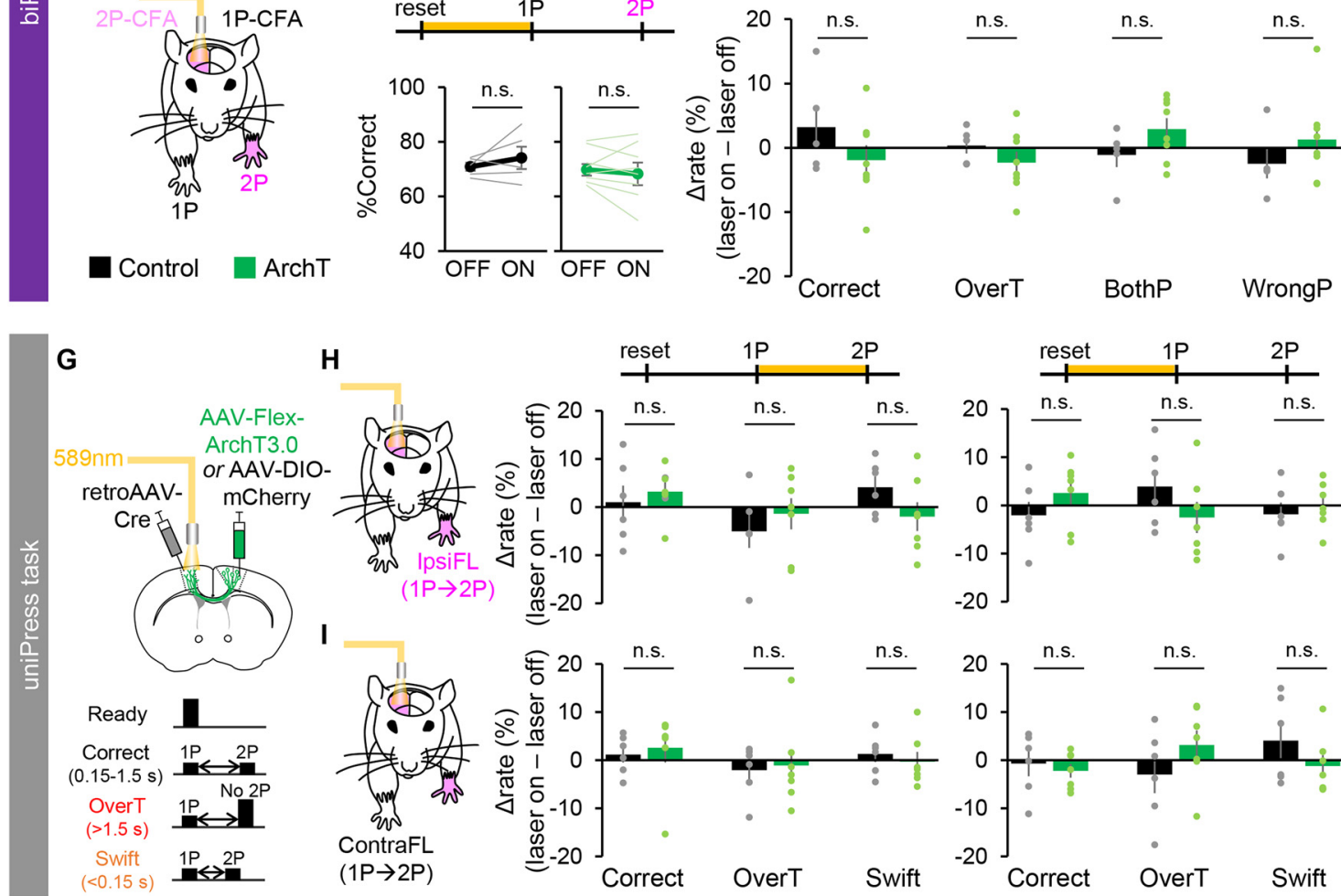

H
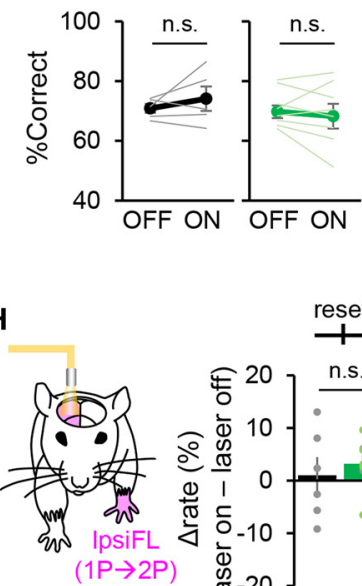

I.
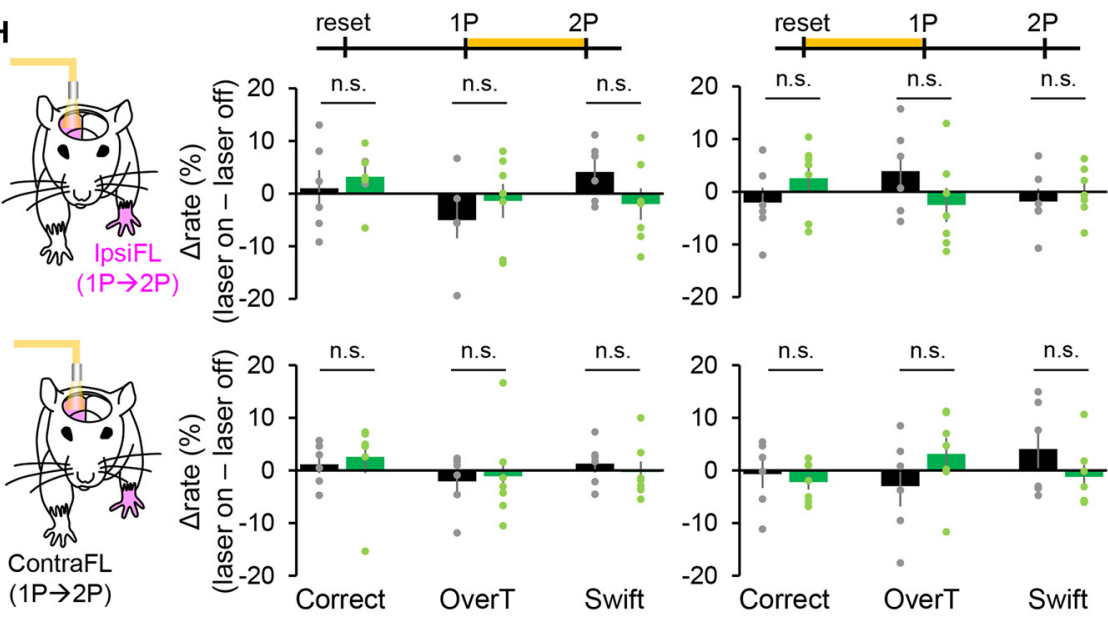

Figure 4. Increased neural activities of corticocortical axon terminals in the 2P-CFA during the 1-2P period involve in correct performance of the biPress task. $\boldsymbol{A}$, Experimental design of a fiber photometry to image the $\mathrm{Ca}^{2+}$ activity of axonal fibers of $1 \mathrm{P}$-CFA neurons in the 2P-CFA. Representative image showing virus-infected CFA neurons and the location of the implanted optic cannula. Scale bars: $500 \mu \mathrm{m}$. B, Representative profiles of axonal $\mathrm{Ca}^{2+}$ dynamics, presented as a heatmap. C, Average $\mathrm{Ca}^{2+}$ dynamics across all mice. Left, Averaged $\mathrm{Ca}^{2+}$ activity in correct and overT incorrect trials. Colored horizontal bars indicate the periods with significantly higher activity compared with incorrect trials. Right, Averaged $\mathrm{Ca}^{2+}$ firing rates at the $2 \mathrm{P}$ timing $([2 \mathrm{P}-250 \mathrm{~ms}, 2 \mathrm{P}+250$ $\mathrm{ms}]$ ). Shaded areas and error bars represent $95 \%$ confidence interval. $\boldsymbol{D}$. Experimental scheme for the photoinhibition of corticocortical projections of 1P-CFA neurons during the biPress task. $\boldsymbol{E}$, Comparisons of the correct rate (left) and differences of rate in correct and each incorrect type trials between optogenetic light off and on (right) by photoinhibition during the interpress interval. $\boldsymbol{F}$, Comparisons of the correct rate (left) and differences of rate in correct and each incorrect type trials between optogenetic light off 
continued

and on (right) by photoinhibition during the pre-1P period. For comparisons of latency to $1 \mathrm{P}$, see Extended Data Figure 4-1A-C. G, Experimental scheme for the photoinhibition of corticocortical projections of CFA neurons during the timed unimanual-press sequence (uniPress) task by either ipsilateral (ipsiFL; $\boldsymbol{H}$ ) or contralateral (contraFL; $\boldsymbol{I}$ ) forelimb to the CFA neurons. 2P was performed by the same forelimb with the 1P. Left, Photoinhibition during the 1-2P period. Right, Photoinhibition during the pre-1P period. All data are represented as mean \pm SEM, and individual data are represented as light-colored lines or circles; ${ }^{*} p<0.05$, ${ }^{* *} p<0.01$, n.s. not significant. For optogenetic inactivation of 1P-CFA neurons, see Extended Data Figure 4-1D-H.

Figure Contributions: Minju Jeong performed all experiments and analyzed the data of optogenetic experiments. Hyeonsu Lee analyzed the data of photometry experiments.

during the pre-1P period did not affect the task performance $(n=5$ mCherry control and $n=8$ ArchT mice, left, two-tailed paired $t$ test, $p=0.393$ for mCherry, $p=0.435$ for ArchT, right, two-tailed unpaired $t$ test, $p=0.220$ for correct, $p=0.291$ for overT, $p=0.153$ for bothP, $p=0.305$ for wrongP; Fig. 4F). This photoinhibition of corticocortical projections also did not interfere with performing $1 \mathrm{P}$ by either contralateral or ipsilateral forelimbs in overall [Extended Data Fig. 4-1A-C; $n=5$ mCherry control and $n=7$ ArchT mice, Mann-Whitney $U$ test, $p=0.26$ (Extended Data Fig. 4-1A, left) and MannWhitney $U$ test, $p=0.432$ (Extended Data Fig. 4-1A, right); $n=5$ mCherry control and $n=7$ ArchT mice, twotailed unpaired $t$ test, $p=0.864$ (Extended Data Fig. 4-1C)], suggesting the interruption of correct $2 \mathrm{P}$ performance without effect on movement execution by optogenetic inactivation during the 1-2P period.

We observed that the optogenetic inactivation of $1 \mathrm{P}-$ CFA neurons during the 1-2P period by photostimulation of Channelrhodopsin 2 (ChR2) expressed in cortical inhibitory interneurons (Zhao et al., 2011; Guo et al., 2014) also significantly reduced the biPress task performance (Extended Data Fig. 4-1D,E, $n=8$ littermate control and $n=8$ VGAT::ChR2 mice, left, Wilcoxon signed-rank test, $p=0.250$ for control, two-tailed paired $t$ test, $p=0.0157$ for VGAT-ChR2; right, two-tailed unpaired $t$ test, $p=$ 0.0103 for correct, $p=0.251$ for overT, $p=0.0109$ for bothP, Mann-Whitney $U$ test, $p=0.195$ for wrongP). However, it did not increase overT trials but increased bothP incorrect trials. It indicates a specific role of corticocortical projecting neurons in transfer of neural activities inducing $2 \mathrm{P}$ after performing 1P. Although further investigation is needed, we assume that this different effect on the type of incorrect trials might be caused because inhibitory interneurons can simultaneously affect throughout the 1P-CFA neurons (Babl et al., 2019) regardless of their encoding information.

To confirm the specific role of corticocortical projections for sequential bimanual movements rather than simply initiation of the next movement sequence, we also tested the optogenetic inactivation effect on sequential unimanual-press task. We expressed the ArchT in one CFA and implanted an optic fiber into the CFA of opposite hemisphere as the previous experiment. Then, we trained mice to sequentially press the same side pedal twice by the single forelimb, which was either contralateral or ipsilateral to the neuronal soma expressing ArchT (Fig. 4G). Mice were water-rewarded for the sequential pedal-press between 0.15 and $1.5 \mathrm{~s}$. They could not be rewarded if they pressed the pedal twice within $0.15 \mathrm{~s}$ (swift) or only once within $1.5 \mathrm{~s}$ (overT). Photoinhibition of corticocortical axon terminals during both $1-2 \mathrm{P}$ and pre-1P periods did not change the unimanual-press task performance [Fig. $4 \mathrm{H}, I, n=6$ mCherry control and $n=7$ ArchT mice, two-tailed unpaired $t$ test, $p=0.572$ for correct, $p=0.459$ for overT, $p=0.140$ for swift (Fig. $4 H$, left) and two-tailed unpaired $t$ test, $p=0.250$ for correct, $p=0.197$ for overT, $p=0.556$ for swift (Fig. $4 H$, right); $n=6$ mCherry control and $n=7$ ArchT mice, left, Mann-Whitney $U$ test, $p=0.366$ for correct, two-tailed unpaired $t$ test, $p=0.837$ for overT, $p=0.541$ for swift, right, two-tailed unpaired $t$ test, $p=0.72$ for correct, $p=0.228$ for overT, $p=0.236$ for swift (Fig. 4/)]. Together, these results further support that the transfer of neural signals via corticocortical projections during movement transition period contributes for coordinating sequential bimanual movements.

\section{Discussion}

The use of left and right limbs with precise sequence and timing is one of the most essential and basic movements to perform various behaviors. Our results show that the neural correlates of sequential bimanual movements can exist within neurons from a single motor cortical hemisphere. In addition, the subsequent transfer of information, presumably to the contralateral cortex through long-range axonal projections (Hagihara et al., 2021), is critical for movement coordination. However, anatomic tracing studies have revealed that many of these efferent neurons contain axons projecting to motor areas such as the striatum and cortex in both hemispheres (Oh et al., 2014; Zingg et al., 2014; Jeong et al., 2016). Therefore, it has been difficult to determine through which cortical pathway information would be transferred to coordinate sequential bimanual movements. Supported by our observation of sequential hemispheric calcium activation patterns from the WFI experiment, we speculate that the motor cortex can coordinate sequential bimanual movements through direct axonal projections to the contralateral motor cortex (Fig. 2). By combining advances in viral tracing and optogenetics, we were able to selectively target the motor cortex-projecting axon terminals of $1 \mathrm{P}$-hemisphere neurons. This approach allows us to specifically examine the corticocortical projections (Fig. 4). Our fiber photometry imaging indicates that information about the $2 \mathrm{P}$ movement is present in these axonal terminals (Fig. 4). Importantly, the inactivation of these axon terminals after $1 \mathrm{P}$, but not before, can impair $2 \mathrm{P}$ movements. The temporal precision of optogenetics allows us to compare the effects of corticocortical axon terminal inactivation before and after $1 \mathrm{P}$ movements, and to 
rule out the possibility that our experimental manipulations merely interfere with movement execution.

To directly correlate sequential bimanual movements with neural activity, we chose the head-fixation approach to minimize motion artifacts while still allowing mice to move during neuro-imaging or extracellular recording. During the design of the behavioral apparatus, we carefully optimized the pedal position so that the mouse can only reach each pedal with a unilateral forelimb. After each press with enough force, the pedals retracted until the next trial to avoid a double-tap confound. Lastly, to separate the neural correlates of bimanual movements from unilateral movements, we mandated sequential right-and-left presses (or vice versa) by rewarding trials where sequential bimanual presses were performed within a narrow time window.

The rodent neocortex contains several neural pathways to facilitate left and right forelimb movements; conversely, a unilateral movement may reflect the contributions of both cortical hemispheres. Indeed, near simultaneous brain-wide activation is typically observed during movement (Makino et al., 2017; Terada et al., 2018; Mayrhofer et al., 2019). Therefore, we suspected that if sequential bimanual movements were mediated by interhemispheric interactions, then bilateral cortical activation patterns may be different for bimanual versus unilateral movements. In bimanual movements alone, we observed that the hemisphere corresponding to the $1 \mathrm{P}$ peaks before the $2 \mathrm{P}$ hemisphere (Fig. 2). This order of activation (1P before 2P) raises the possibility that information is propagating from the $1 \mathrm{P}$ to the $2 \mathrm{P}$ hemisphere; however, we also considered the alternative premise that each cortex operates independently of the other (Li et al., 2016). In particular, we examined two additional possibilities: (1) the de novo generation of movement signals independently in each hemisphere (without interactions); and (2) that a central upstream brain region transmits sequential information to the left and right hemispheres.

These alternative possibilities generate testable hypotheses. For example, if movement signals were indeed independently generated within each hemisphere, we would not expect to see any signals corresponding to the $2 \mathrm{P}$ movement within the 1P hemisphere. However, our extracellular recording characterization of $1 \mathrm{P}$-hemisphere neurons identifies several classes of neurons that show increased firing rates during $1 \mathrm{P}$ movements, $2 \mathrm{P}$ movements, or even during both $1 \mathrm{P}$ and $2 \mathrm{P}$ movements simultaneously (Extended Data Fig. 3-1B,C). Meanwhile, if a central, upstream controller transmitted information to both $1 \mathrm{P}$ and $2 \mathrm{P}$ hemispheres, then inactivating neurons in the 1P-hemisphere would not affect the number of $2 \mathrm{P}$ movements. However, our results show that 1P-hemisphere inactivation after 1P movement, but not before, significantly reduces the number of $2 \mathrm{P}$ movements (Extended Data Fig. 4-1D-H). Both of alternative scenarios are on the premise that the $1 \mathrm{P}$ and $2 \mathrm{P}$ hemispheres independently control each left and right forelimb, and thus, perturbing $1 \mathrm{P}$-hemisphere should have no repercussion on the subsequent $2 \mathrm{P}$ forelimb movement.

We also appraised several important caveats of our experiments. For example, the presence of bilateral projecting cortical neurons suggest the possibility that performing $2 \mathrm{P}$ by the ipsilateral forelimb to the $1 \mathrm{P}-\mathrm{CFA}$ would be generated from the contributions of 1P-CFA neurons besides 2P-CFA (Soma et al., 2017). If so then, the neural activity of bothP incorrect trials before $1 \mathrm{P}$ are supposed to be the same or higher than overT and correct trials since both contralateral and ipsilateral forelimbs are involved. However, our result showed the opposite: activity of bothP was significantly lower than correct trials (Extended Data Fig. 3-1D). Moreover, the neural activities of bothP and wrongP incorrect trials already declined after the onset of movement (MovS), while activity of overT trials sharply dropped only during the 1-2P period (Fig. 3B). Therefore, it is difficult to interpret our results may simply be the activity of neurons in the 1P-hemisphere representing ipsilateral forelimb movements for 2P. Consistently, our optogenetic inactivation results also cannot be explained as the inherent interference with ipsilateral movement execution because inactivating neurons in the CFA did not affect ipsilateral forelimb movements performing 1P (Extended Data Fig. 4-1C,H) and 2P (Fig. 4H).

Our data that optogenetic inactivation of corticocortical projections did not alter the performance of unimanualpress sequence task suggest that corticocortical projections may not be involved in simple sequence movements. Since this unimanual sequence task did not require movement interaction between contralateral and ipsilateral forelimbs, it might not require the transfer of motor signals to the opposite cortical hemisphere but rather the transfer to the striatum (Eliassen et al., 1999; Jin et al., 2014; Rothwell et al., 2015; Geddes et al., 2018). Of note, it may be a concern to use the ArchT for axon terminal inhibition because a previous study has observed a paradoxical rebound of the increase of terminal neurotransmitter releases after ArchT activation (Mahn et al., 2016). However, it is caused only after the long-term illumination, but not after short-term illumination protocol (Manita et al., 2015; El-Gaby et al., 2016; Mahn et al., 2016; Ozawa et al., 2017; Uematsu et al., 2017) that we used in this study (1.5 s at most). Thus, we believe that the major effects of our manipulation in this study is reducing, not increasing, the axonal release of neurotransmitter.

In conclusion, our results support a model of direct corticocortical communication to enable the orchestration of sequential bimanual movements. Importantly, while our results highlight the role of corticocortical projections, this does not mean that other axonal projections or other brain regions are unnecessary. It may be valuable to identify downstream regions where information coordinating fine sequential bimanual movements are integrated, processed, and transmitted. To this end, we hope that our newly developed headfixed paradigm cannot only further support this line of research, but also be broadly applied to other behavioral experiments beyond the motor function involving decisionmaking and attentional set shifting.

\section{References}

Babl SS, Rummell BP, Sigurdsson T (2019) The spatial extent of optogenetic silencing in transgenic mice expressing channelrhodopsin in inhibitory interneurons. Cell Rep 29:1381-1395.e4. 
Bloom JS, Hynd GW (2005) The role of the corpus callosum in interhemispheric transfer of information: excitation or inhibition? Neuropsychol Rev 15:59-71.

Bonzano L, Tacchino A, Roccatagliata L, Abbruzzese G, Mancardi GL, Bove M (2008) Callosal contributions to simultaneous bimanual finger movements. J Neurosci 28:3227-3233.

Diedrichsen J, Kornysheva K (2015) Motor skill learning between selection and execution. Trends Cogn Sci 19:227-233.

Eliassen JC, Baynes K, Gazzaniga MS (1999) Direction information coordinated via the posterior third of the corpus callosum during bimanual movements. Exp Brain Res 128:573-577.

El-Gaby M, Zhang Y, Wolf K, Schwiening CJ, Paulsen O, Shipton OA (2016) Archaerhodopsin selectively and reversibly silences synaptic transmission through altered pH. Cell Rep 16:2259-2268.

Geddes CE, Li H, Jin X (2018) Optogenetic editing reveals the hierarchical organization of learned action sequences. Cell 174:32-43 e15.

Gooijers J, Swinnen SP (2014) Interactions between brain structure and behavior: the corpus callosum and bimanual coordination. Neurosci Biobehav Rev 43:1-19.

Guo ZV, Li N, Huber D, Ophir E, Gutnisky D, Ting JT, Feng G, Svoboda K (2014) Flow of cortical activity underlying a tactile decision in mice. Neuron 81:179-194.

Hagihara KM, Ishikawa AW, Yoshimura Y, Tagawa Y, Ohki K (2021) Long-range interhemispheric projection neurons show biased response properties and fine-scale local subnetworks in mouse visual cortex. Cereb Cortex 31:1307-1315.

Hira R, Ohkubo F, Ozawa K, Isomura Y, Kitamura K, Kano M, Kasai $\mathrm{H}$, Matsuzaki M (2013) Spatiotemporal dynamics of functional clusters of neurons in the mouse motor cortex during a voluntary movement. J Neurosci 33:1377-1390.

Jeong M, Kim Y, Kim J, Ferrante DD, Mitra PP, Osten P, Kim D (2016) Comparative three-dimensional connectome map of motor cortical projections in the mouse brain. Sci Rep 6:20072.

Jin X, Tecuapetla F, Costa RM (2014) Basal ganglia subcircuits distinctively encode the parsing and concatenation of action sequences. Nat Neurosci 17:423-430.

Kennerley SW, Diedrichsen J, Hazeltine E, Semjen A, Ivry RB (2002) Callosotomy patients exhibit temporal uncoupling during continuous bimanual movements. Nat Neurosci 5:376-381.

Kim CK, Yang SJ, Pichamoorthy N, Young NP, Kauvar I, Jennings JH, Lerner TN, Berndt A, Lee SY, Ramakrishnan C, Davidson TJ, Inoue M, Bito H, Deisseroth K (2016) Simultaneous fast measurement of circuit dynamics at multiple sites across the mammalian brain. Nat Methods 13:325-328.

Kim J, Kim Y, Nakajima R, Shin A, Jeong M, Park AH, Jeong Y, Jo S, Yang S, Park H, Cho SH, Cho KH, Shim I, Chung JH, Paik SB, Augustine GJ, Kim D (2017) Inhibitory basal ganglia inputs induce excitatory motor signals in the thalamus. Neuron 95:1181-1196. e8.

Lashley KS (1951) The problem of serial order in behavior. Oxford: Bobbs-Merrill.

Lee SH, Kwan AC, Zhang S, Phoumthipphavong V, Flannery JG, Masmanidis SC, Taniguchi H, Huang ZJ, Zhang F, Boyden ES, Deisseroth K, Dan Y (2012) Activation of specific interneurons improves V1 feature selectivity and visual perception. Nature 488:379-383.

Lemon RN (2008) Descending pathways in motor control. Annu Rev Neurosci 31:195-218.

Li N, Daie K, Svoboda K, Druckmann S (2016) Robust neuronal dynamics in premotor cortex during motor planning. Nature 532:459-464.

Lim BK, Huang KW, Grueter BA, Rothwell PE, Malenka RC (2012) Anhedonia requires MC4R-mediated synaptic adaptations in nucleus accumbens. Nature 487:183-189.
Mahn M, Prigge M, Ron S, Levy R, Yizhar O (2016) Biophysical constraints of optogenetic inhibition at presynaptic terminals. Nat Neurosci 19:554-556.

Makino H, Ren C, Liu H, Kim AN, Kondapaneni N, Liu X, Kuzum D, Komiyama T (2017) Transformation of cortex-wide emergent properties during motor learning. Neuron 94:880-890.e8.

Manita S, Suzuki T, Homma C, Matsumoto T, Odagawa M, Yamada K, Ota K, Matsubara C, Inutsuka A, Sato M, Ohkura M, Yamanaka A, Yanagawa Y, Nakai J, Hayashi Y, Larkum ME, Murayama M (2015) Atop-down cortical circuit for accurate sensory perception. Neuron 86:1304-1316.

Mayrhofer JM, El-Boustani S, Foustoukos G, Auffret M, Tamura K, Petersen CCH (2019) Distinct contributions of whisker sensory cortex and tongue-jaw motor cortex in a goal-directed sensorimotor transformation. Neuron 103:1034-1043.e4.

Oh SW, Harris JA, Ng L, Winslow B, Cain N, Mihalas S, Wang Q, Lau C, Kuan L, Henry AM, Mortrud MT, Ouellette B, Nguyen TN, Sorensen SA, Slaughterbeck CR, Wakeman W, Li Y, Feng D, Ho A, Nicholas E, et al. (2014) A mesoscale connectome of the mouse brain. Nature 508:207-214.

Ozawa T, Ycu EA, Kumar A, Yeh LF, Ahmed T, Koivumaa J, Johansen JP (2017) A feedback neural circuit for calibrating aversive memory strength. Nat Neurosci 20:90-97.

Penfield W, Rasmussen T (1950) The cerebral cortex of man; a clinical study of localization of function. New York: MacMillan.

Rothwell PE, Hayton SJ, Sun GL, Fuccillo MV, Lim BK, Malenka RC (2015) Input- and output-specific regulation of serial order performance by corticostriatal circuits. Neuron 88:345-356.

Soma S, Saiki A, Yoshida J, Ríos A, Kawabata M, Sakai Y, Isomura Y (2017) Distinct laterality in forelimb-movement representations of rat primary and secondary motor cortical neurons with intratelencephalic and pyramidal tract projections. J Neurosci 37:1090410916.

Tanji J, Okano K, Sato KC (1988) Neuronal activity in cortical motor areas related to ipsilateral, contralateral, and bilateral digit movements of the monkey. J Neurophysiol 60:325-343.

Tennant KA, Adkins DL, Donlan NA, Asay AL, Thomas N, Kleim JA, Jones TA (2011) The organization of the forelimb representation of the C57BL/6 mouse motor cortex as defined by intracortical microstimulation and cytoarchitecture. Cereb Cortex 21:865-876.

Terada SI, Kobayashi K, Ohkura M, Nakai J, Matsuzaki M (2018) Super-wide-field two-photon imaging with a micro-optical device moving in post-objective space. Nat Commun 9:3550.

Uematsu A, Tan BZ, Ycu EA, Cuevas JS, Koivumaa J, Junyent F, Kremer EJ, Witten IB, Deisseroth K, Johansen JP (2017) Modular organization of the brainstem noradrenaline system coordinates opposing learning states. Nat Neurosci 20:1602-1611.

Wahl M, Lauterbach-Soon B, Hattingen E, Jung P, Singer O, Volz S, Klein JC, Steinmetz H, Ziemann U (2007) Human motor corpus callosum: topography, somatotopy, and link between microstructure and function. J Neurosci 27:12132-12138.

Wekselblatt JB, Flister ED, Piscopo DM, Niell CM (2016) Large-scale imaging of cortical dynamics during sensory perception and behavior. J Neurophysiol 115:2852-2866.

Zhao S, Ting JT, Atallah HE, Qiu L, Tan J, Gloss B, Augustine GJ, Deisseroth K, Luo M, Graybiel AM, Feng G (2011) Cell type-specific channelrhodopsin-2 transgenic mice for optogenetic dissection of neural circuitry function. Nat Methods 8:745-752.

Zhou J, Wen Y, She L, Sui YN, Liu L, Richards LJ, Poo MM (2013) Axon position within the corpus callosum determines contralateral cortical projection. Proc Natl Acad Sci USA 110:E2714-E2723.

Zingg B, Hintiryan H, Gou L, Song MY, Bay M, Bienkowski MS, Foster NN, Yamashita S, Bowman I, Toga AW, Dong HW (2014) Neural networks of the mouse neocortex. Cell 156:1096-1111. 